\title{
Error estimates of implicit Runge-Kutta methods for quasilinear abstract equations of parabolic type in Banach spaces
}

Dedicated to Professor Kyûya Masuda on the occasion of his 60th birthday

\author{
By Etsushi NaKaguCHI and Atsushi YagI \\ (Received May 8, 1997) \\ (Revised September 29, 1997) \\ (from Journal of the Mathematical Society of Japan)
}

\section{Introduction}

In the paper Lubich and Ostermann [15], they have shown that the RungeKutta approximation methods are useful even for the ordinary differential equations in an infinite dimensional space. They studied the convergence properties of implicit Runge-Kutta methods applied to the initial value problem of the quasilinear abstract equation

$$
\left\{\begin{array}{l}
\frac{d u}{d t}+A(u) u=f(u), \quad 0<t \leq T \\
u(0)=u_{0}
\end{array}\right.
$$

of parabolic type. In the paper [15] the framework was set in a triple of Hilbert spaces $V \subset H=H^{\prime} \subset V^{\prime}$, that is, $A(u)$ are defined for $u \in V$ and each $A(u)$ is associated with a continuous bilinear form on $V$ satisfying the Gårding inequality and therefore is a bounded operator from $V$ into $V^{\prime}$ and a densely defined, closed linear operator in $H$. In this framework the solution $u$ to $(\mathrm{E})$ is constructed in the space $L^{2}((0, T) ; V) \cap \mathcal{C}([0, T] ; H) \cap H^{1}\left((0, T) ; V^{\prime}\right)$. Consequently the error estimates are also obtained in the same function space.

It is known, however, that the problem (E) can be considered in a Banach space $X$ under the situation where each $-A(u)$ is the generator of an analytic semigroup on $X$. Also it is known that somewhat stronger results are obtained in this setting. In fact, under suitable assumptions on $A(u), f(u)$ and $u_{0}$, a regular solution $u$ in the space $\mathcal{C}^{1}([0, T] ; X) \cap \mathcal{C}\left([0, T] ; \mathcal{D}\left(A\left(u_{0}\right)\right)\right.$ ) (where $\mathcal{D}\left(A\left(u_{0}\right)\right)$ is the domain of the operator $A\left(u_{0}\right)$ equipped with the graph norm) can be constructed, see Sobolevskii [25] and Lunardi [16], cf. also [33]. 
Therefore we thought that it might be meaningful to study similar convergence properties of implicit Runge-Kutta methods in the framework of Banach space to obtain stronger error estimates in the space $\mathcal{C}\left([0, T] ; \mathcal{D}\left(A\left(u_{0}\right)\right)\right)$.

The domains $\mathcal{D}(A(u)) \equiv \mathcal{D}\left(A\left(u_{0}\right)\right)$ of $A(u)$ are independent of the unknowns $u$. The spectral sets $\sigma(A(u))$ of $A(u)$ are assumed to be subsets of some closed sectorial region $\overline{S_{\varphi}}, 0<\varphi<\pi / 2$, where $S_{\varphi}=\{z \in \mathbb{C} ;|\arg z|<\varphi\}$. The RungeKutta methods are assumed to be strongly $A(\theta)$-stable with $\varphi<\theta \leq \pi / 2$. In Section 2 basic properties of the stability function of strongly $A(\theta)$-stable scheme will be summarized.

In Section 3 we shall consider the autonomous linear equation in $X$ with a coefficient operator $A$. As shown in Crouzeix et al. [5] and Palencia [19], there exists a family of bounded operators which are given by the functional calculus of $A$ with respect to the stability function and which describe the approximate solutions to the autonomous linear problem. Moreover, in this paper we shall show that the bounded operators enjoy properties quite analogous to the analytic semigroup generated by $-A$, see Proposition 3.4.

In Section 4 the non autonomous linear equation will be studied. Our techniques are quite different from [15] in which the energy estimates are the main tools. Instead, a family of bounded operators will be introduced as a fundamental solution for constructing the approximate solutions. This family of bounded operators enjoys properties quite analogous to the fundamental solution of the continuous non autonomous problem, and consequently provides a new formula for describing the approximate solutions of the nonlinear problem (E).

By virtue of these representations, stronger results on the stability and on the convergence of the approximate solutions to (E) will be established in Section 5 . Our results are applicable to the various quasilinear parabolic systems. We consider, as an example, the Keller-Segel equations appearing in mathematical biology.

The authors believe the obtained representation formula is useful even in the study of the convergence property in spatial discretizations. But this will be studied in the forthcoming paper. For the linear equations there is a lot of literature on this problem. We quote Helfrich [8] and Fujita and Mizutani [6] as the pioneering researches. In [8] the autonomous linear equation in a Hilbert space is considered, the coefficient being a self adjoint operator; and the error estimate is obtained by means of the Ritz operator. This result is then generalized by Ushijima $[29,30]$ and Piskarev [2] to the framework of Banach space. In [6] the autonomous linear equation in $L^{2}$ space is considered, and the rate of convergence of the finite element method is obtained. This result is extended subsequently by Suzuki [26, 27] for the non autonomous linear equation. We quote also researches using somewhat different techniques, Bramble et al. [3], Baker et al. [1], and Sammon [22, 23]. For the full list, we refer the reader to References in Fujita and Suzuki [7]. For the semilinear equation we quote Keeling [10]. 
We conclude this section by recalling some terminologies for numerical analysis used in this paper, cf. $[4,9]$.

An $s$-stage Runge-Kutta method applied to the initial value problem of an ordinary differential equation

$$
\left\{\begin{array}{l}
\frac{d u}{d t}=F(t, u), \quad 0 \leq t \leq T \\
u(0)=u_{0}
\end{array}\right.
$$

with a stepsize $h>0$ is written as

$$
\begin{aligned}
U_{n+1} & =U_{n}+h \sum_{i=1}^{s} b_{i} F\left(t_{n}+c_{i} h, V_{n, i}\right), \quad n=0,1, \ldots, N \\
V_{n, i} & =U_{n}+h \sum_{j=1}^{s} a_{i j} F\left(t_{n}+c_{j} h, V_{n, j}\right), \quad i=1, \ldots, s, \\
U_{0} & =u_{0},
\end{aligned}
$$

where $t_{n}=n h$ and $t_{N+1}=(N+1) h \leq T$. The approximate solution $\left\{U_{n}\right\}$ is given recursively by $(1.2 \mathrm{a})$ with $\left\{V_{n, i}\right\}$ given by $(1.2 \mathrm{~b})$ at every time step $n$. The parameters $a_{i j}, b_{i}, c_{i}$ are all given real numbers. Using the matrix notation $\mathcal{A}=\left[a_{i j} ; i, j=1, \ldots, s\right], \boldsymbol{b}=\left[b_{1}, \ldots, b_{s}\right]^{T}, \boldsymbol{e}=[1, \ldots, 1]^{T}$ and $\mathcal{C}=\operatorname{diag}\left[c_{1}, \ldots, c_{s}\right]$, we can rewrite $(1.2 \mathrm{a}-\mathrm{c})$ as

$$
\left\{\begin{aligned}
U_{n+1} & =U_{n}+h \boldsymbol{b} \boldsymbol{F}_{n}\left(\boldsymbol{V}_{n}\right), \quad n=0,1, \ldots, N, \\
\boldsymbol{V}_{n} & =\boldsymbol{e} U_{n}+h \mathcal{A} \boldsymbol{F}_{n}\left(\boldsymbol{V}_{n}\right), \\
U_{0} & =u_{0}
\end{aligned}\right.
$$

here $\boldsymbol{V}_{n}=\left[V_{n, 1}, \ldots, V_{n, s}\right]^{T}$ and $\boldsymbol{F}_{n}(\boldsymbol{W})=\left[F\left(t_{n}+h c_{1}, W_{1}\right), \ldots, F\left(t_{n}+h c_{s}, W_{s}\right)\right]^{T}$ with $\boldsymbol{W}=\left[W_{1}, \ldots, W_{s}\right]^{T}$.

The Runge-Kutta scheme (1.3) is said to have an order of accuracy $p$, or briefly to be of order $p$, if $U_{n+1}-u\left(t_{n+1}\right)=O\left(h^{p+1}\right)$ as $h \rightarrow 0$ when $U_{n}=u\left(t_{n}\right)$. By Butcher an equivalent condition for this has been given in terms of $\{\mathcal{A}, \boldsymbol{b}, \mathcal{C}\}$, see [4, Theorem 307B].

Theorem (Butcher's order condition). Let $c_{i}=\sum_{j=1}^{s} a_{i j}$ for all $i=$ $1, \ldots, s$. Then the Runge-Kutta scheme (1.3) is of order $p$ if and only if the coeffcient set $\{\mathcal{A}, \boldsymbol{b}, \mathcal{C}\}$ satisfies

$$
\gamma(\tau) \Phi(\tau)=1
$$


for each rooted tree $\tau$ which has vertices no more than $p$. Here, $\gamma(\tau)$ denotes the density of the tree $\tau$ and $\Phi(\tau)$ the elementary weight for $\tau$ with respect to the scheme.

In addition, the stage order and the quadrature order of the Runge-Kutta scheme (1.3) are defined respectively by the maximum of numbers $q$ such that

$$
\mathcal{A C}^{k-1} \boldsymbol{e}=\frac{1}{k} \mathcal{C}^{k} \boldsymbol{e}, \quad \text { for all } k=1, \ldots, q,
$$

and by the maximum of numbers $r$ such that

$$
\boldsymbol{b}^{T} \mathcal{C}^{k-1} \boldsymbol{e}=\frac{1}{k}, \quad \text { for all } k=1, \ldots, r
$$

It is known that the relation

$$
\min \{q+1, r\} \leq p \leq \min \{2 s, r\}
$$

holds [4, pp.218-219 and 289].

The Runge-Kutta scheme is said to be explicit if $a_{i j}=0$ for $1 \leq i \leq j \leq s$ and implicit otherwise.

The scheme is said to be $A(\theta)$-stable if $(\mathcal{I}+z \mathcal{A})^{-1}$ is holomorphic on a domain containing $\overline{S_{\theta}}, 0<\theta \leq \pi / 2$, where $S_{\theta}=\{z \in \mathbb{C} ;|\arg z|<\theta\}$, and if the stability function $R(z)=1-z \boldsymbol{b}^{T}(\mathcal{I}+z \mathcal{A})^{-1} \boldsymbol{e}$ satisfies

$$
|R(z)| \leq 1, \quad z \in \overline{S_{\theta}} .
$$

The scheme is storngly $A(\theta)$-stable if it is $A(\theta)$-stable and if the limit $R(\infty)=$ $1-\boldsymbol{b}^{T} \mathcal{A}^{-1} \boldsymbol{e}$ exists with the estimate

$$
|R(\infty)|<1
$$

Notice that explicit Runge-Kutta methods can not be $A(\theta)$-stable, because stability functions

$$
R(z)=1-z \boldsymbol{b}^{T}(\mathcal{I}+z \mathcal{A})^{-1} \boldsymbol{e}=1-z \boldsymbol{b}^{T} \sum_{k=0}^{s-1}(-1)^{k} z^{k} \mathcal{A}^{k} \boldsymbol{e}
$$

are all polynomials of degree at most $s$ and (1.7) never holds.

Other notations. $X$ denotes a Banach space, its norm is denoted by $\|\cdot\|_{X}$. The $s$-ple product space $X^{s}$ of $X$ consisting of column vectors $[\cdot, \ldots, \cdot]^{T}$ is denoted by $\boldsymbol{X}, \boldsymbol{X}$ is equipped with the usual product norm. 
By a matrix of linear operators acting in $X$

$$
\boldsymbol{A}=\left[\begin{array}{ccc}
A_{11} & \cdots & A_{1 s} \\
\vdots & \ddots & \vdots \\
A_{s 1} & \cdots & A_{s s}
\end{array}\right]
$$

we denote a linear operator acting in $\boldsymbol{X}$ in the usual manner. Similarly, by a column vector and a row vector of linear operators in $X$

$$
\boldsymbol{B}=\left[\begin{array}{c}
B_{1} \\
\vdots \\
B_{s}
\end{array}\right], \quad \boldsymbol{C}=\left[\begin{array}{lll}
C_{1} & \cdots & C_{s}
\end{array}\right]
$$

we denote an operator from the domain $\mathcal{D}(\boldsymbol{B}) \subset X$ into $\boldsymbol{X}$ and an operator from the domain $\mathcal{D}(\boldsymbol{C}) \subset \boldsymbol{X}$ into $X$, respectively.

We define also the products of such operators. More precisely, for $\boldsymbol{A}, \boldsymbol{B}, \boldsymbol{C}$ as above and an operator $\widetilde{\boldsymbol{A}}=\left[\widetilde{A}_{i j}\right]$ in $\boldsymbol{X}$, we define

$$
\begin{aligned}
\boldsymbol{A} \tilde{\boldsymbol{A}} & =\left[\begin{array}{ccc}
A_{11} \widetilde{A}_{11}+\cdots+A_{1 s} \widetilde{A}_{s 1} & \cdots & A_{11} \widetilde{A}_{1 s}+\cdots+A_{1 s} \widetilde{A}_{s s} \\
\vdots & \ddots & \vdots \\
A_{s 1} \widetilde{A}_{11}+\cdots+A_{s s} \widetilde{A}_{s 1} & \cdots & A_{s 1} \widetilde{A}_{1 s}+\cdots+A_{s s} \widetilde{A}_{s s}
\end{array}\right], \\
\boldsymbol{A} \boldsymbol{B} & =\left[\begin{array}{c}
A_{11} B_{1}+\cdots+A_{1 s} B_{s} \\
\vdots \\
A_{s 1} B_{1}+\cdots+A_{s s} B_{s}
\end{array}\right], \\
\boldsymbol{C A} & =\left[\begin{array}{lll}
C_{1} A_{11}+\cdots+C_{s} A_{s 1} & \cdots & C_{1} A_{1 s}+\cdots+C_{s} A_{s s}
\end{array}\right], \\
\boldsymbol{C B} & =C_{1} B_{1}+\cdots+C_{s} B_{s} .
\end{aligned}
$$

If there is no fear of confusion, a matrix $\mathcal{M}=\left[m_{i j} ; i, j=1, \ldots, s\right]$, a column vector $\boldsymbol{k}=\left[k_{1}, \ldots, k_{s}\right]^{T}$ and a row vector $\boldsymbol{l}=\left[l_{1}, \ldots, l_{s}\right]$ are identified with the bounded operator $\left[m_{i j} I\right] \in \mathcal{L}(\boldsymbol{X})$, the bounded operator $\left[k_{j} I\right]^{T} \in \mathcal{L}(X, X)$ and the bounded operator $\left[l_{i} I\right] \in \mathcal{L}(\boldsymbol{X}, X)$, respectively, $I$ being the identity operator of $X$. The identity matrix of order $s$ is denoted by $\mathcal{I}$.

Throughout this paper we denote by $C$ the generic constant determined in each occurrence by the initial constants appearing in the assumptions. In a case when $C$ depends on some parameter, say $\zeta$, it will be denoted by $C_{\zeta}$.

Acknowledgement. The authors express their hearty thanks to Professor S. Piskarev; discussions with him were very valuable for understanding the problems. They also thank the referee very much for useful comments, especially for bringing important references to their attention. 


\section{Some properties of the stability functions of Runge-Kutta methods}

Let $R(z)$ be the stability function of a strongly $A(\theta)$-stable Runge-Kutta scheme. This section is devoted to surveying basic properties of $R(z)$, which are used in the subsequent sections. Essential parts of the results are seen in [5].

As already noticed, $R(z)$ is a holomorphic function in a domain containing $\overline{S_{\theta}}$. More precisely we observe the following proposition.

Proposition 2.1. Singular points of $R(z)$ are contained in the closed domain

$$
\Sigma=\left\{z \in \mathbb{C} ; \frac{1}{\|\mathcal{A}\|} \leq|z| \leq\left\|\mathcal{A}^{-1}\right\|,|\arg z| \geq \theta\right\}
$$

Proof. By definition, the singular points of $R(z)$ are the same as those of $(\mathcal{I}+z \mathcal{A})^{-1}$. It is clear that, if $|z|\|\mathcal{A}\|<1,(\mathcal{I}+z \mathcal{A})^{-1}$ is holomorphic. On the other hand, since $(\mathcal{I}+z \mathcal{A})^{-1}=\mathcal{A}^{-1}\left(z+\mathcal{A}^{-1}\right)^{-1}$, if $|z|>\left\|\mathcal{A}^{-1}\right\|,(\mathcal{I}+z \mathcal{A})^{-1}$ is holomorphic.

Since $\lim _{z \rightarrow \infty} z(\mathcal{I}+z \mathcal{A})^{-1}=\mathcal{I}$, we obtain that $R(\infty)=1-\boldsymbol{b}^{T} \mathcal{A}^{-1} \boldsymbol{e}$. Therefore,

$$
R(z)-R(\infty)=\boldsymbol{b}^{T} \mathcal{A}^{-1}(\mathcal{I}+z \mathcal{A})^{-1} \boldsymbol{e}
$$

In addition, we have:

$$
|R(z)-R(\infty)| \leq \frac{C}{|z|-\left\|\mathcal{A}^{-1}\right\|}
$$

Since $|R(\infty)|<1$, there exists some $0<\kappa<1$ such that

$$
|R(z)| \leq \kappa, \quad|z| \geq \delta^{-1}
$$

if $\delta>0$ is sufficiently small.

Since

$$
R(z)=1-z \boldsymbol{b}^{T} \boldsymbol{e}+z^{2} \boldsymbol{b}^{T} \mathcal{A}(\mathcal{I}+z \mathcal{A})^{-1} \boldsymbol{e}
$$

the stability condition (1.7) implies that $b=b^{T} \cdot e \geq 0$. On the other hand, from (2.4) we see that

$$
R(z)-e^{-b z}=O\left(|z|^{2}\right)
$$

in a neighborhood of $z=0$. Furthermore we see that

$$
|R(z)| \leq e^{C|z|^{2}}\left|e^{-b z}\right|
$$


in a neighborhood of the origin. Since $\left|e^{-b z}\right| \leq e^{-b \cos \theta|z|}$ if $|\arg z| \leq \theta$, we can conclude that there exist some $\nu, \eta>0$ such that

$$
\begin{aligned}
& |R(z)| \leq e^{-\nu|z|}, \quad|\arg z| \leq \theta,|z| \leq \delta \\
& |R(z)| \leq e^{\eta|z|}, \quad|\arg z|>\theta,|z| \leq \delta
\end{aligned}
$$

if $\delta>0$ is sufficiently small.

Fix a sufficiently small $\delta>0$ so that (2.3), (2.5) and (2.6) hold, and consider the domain encircled by the contour

$$
\{|z|=\delta,|\arg z| \leq \theta\} \cup\left\{\delta \leq|z| \leq \delta^{-1},|\arg z|=\theta\right\} \cup\left\{|z|=\delta^{-1},|\arg z| \leq \theta\right\} .
$$

In this domain $R(z)$ is holomorphic with the estimate $|R(z)| \leq 1$. Then the maximum principle implies that for any $0<\theta^{\prime}<\theta$, there exists some $0<\kappa^{\prime}<1$ such that

$$
|R(z)| \leq \kappa^{\prime}, \quad \delta \leq|z| \leq \delta^{-1},|\arg z| \leq \theta^{\prime}
$$

\section{Autonomous linear equations}

In this section we consider an implicit Runge-Kutta (R-K) method for the Cauchy problem of an autonomous linear evolution equation

$$
\left\{\begin{array}{l}
\frac{d u}{d t}+A u=f(t), \quad 0<t \leq T \\
u(0)=u_{0}
\end{array}\right.
$$

in a Banach space $X$. Here, $-A$ is the infinitesimal generator of an analytic semigroup on $X, f$ is an $X$-valued function defined on $[0, T]$, and $u_{0} \in X$ is an initial value.

We shall assume the following conditions.

(A1) The resolvent set $\rho(A)$ contains a sector $\mathbb{C} \backslash \overline{S_{\varphi}}$ for some $\varphi, 0<\varphi<\pi / 2$, and the resolvent satisfies an estimate

$$
\left\|(\lambda-A)^{-1}\right\|_{\mathcal{L}(X)} \leq \frac{M}{|\lambda|+1}, \quad \lambda \notin \overline{S_{\varphi}}
$$

with some constant $M$.

(F1) For a number $\sigma>0, f \in \mathcal{C}^{\sigma}([0, T] ; X)$.

(I1) $u_{0} \in \mathcal{D}(A)$.

For the scheme we assume the following. 
(RK1) The scheme is of order $p$ and has a stage order $q$ with $1 \leq q+1 \leq p$.

(RK2) The scheme is strongly $A(\theta)$-stable with $\varphi<\theta \leq \pi / 2$.

Let $0<h \leq T$ and let $N \geq 0$ be an integer such that $(N+1) h \leq T$. As the $s$-stage R-K scheme applied to (3.1), we obtain the following approximation solution

$$
\left\{\begin{aligned}
U_{n+1} & =U_{n}+h \boldsymbol{b}^{T}\left\{-A \boldsymbol{V}_{n}+\boldsymbol{f}\left(\boldsymbol{\tau}_{n}\right)\right\}, \quad n=0,1, \ldots, N \\
\boldsymbol{V}_{n} & =\boldsymbol{e} U_{n}+h \mathcal{A}\left\{-A \boldsymbol{V}_{n}+\boldsymbol{f}\left(\boldsymbol{\tau}_{n}\right)\right\}, \\
U_{0} & =u_{0},
\end{aligned}\right.
$$

where $\boldsymbol{f}\left(\boldsymbol{\tau}_{n}\right)=\left[f\left(t_{n}+c_{1} h\right), \ldots, f\left(t_{n}+c_{s} h\right)\right]^{T}, \boldsymbol{\tau}_{n}=\left(t_{n} \mathcal{I}+h \mathcal{C}\right) \boldsymbol{e}=\left[t_{n}+c_{1} h, \ldots, t_{n}+\right.$ $\left.c_{s} h\right]^{T}$.

As will be proved below by Lemma $3.1, \mathcal{I}+h \mathcal{A} A$ has the bounded inverse on $X^{s}$ for each $h$. Then (3.3) is written as

$$
\left\{\begin{aligned}
U_{n+1} & =U_{n}+h \boldsymbol{b}^{T}\left\{-A \boldsymbol{V}_{n}+\boldsymbol{f}\left(\boldsymbol{\tau}_{n}\right)\right\} \\
\boldsymbol{V}_{n} & =\boldsymbol{J}_{h}\left\{\boldsymbol{e} U_{n}+h \mathcal{A} \boldsymbol{f}\left(\boldsymbol{\tau}_{n}\right)\right\} \\
U_{0} & =u_{0}
\end{aligned}\right.
$$

where $\boldsymbol{J}_{h}=(\mathcal{I}+h \mathcal{A} A)^{-1}$.

Therefore, if we introduce the notation

$$
R(h A)=1-h b^{T} A J_{h} e
$$

the solution $U_{n}$ is given by the formula

$$
U_{n}=R(h A)^{n} u_{0}+h \sum_{l=0}^{n-1} R(h A)^{n-l-1} \boldsymbol{b}^{T} \boldsymbol{J}_{h} \boldsymbol{f}\left(\tau_{l}\right), \quad n=0,1, \ldots, N+1
$$

LEMma 3.1. The inverse of $\mathcal{I}+h \mathcal{A} A$ exists as a bounded operator on $X^{s}$. Moreover, $\boldsymbol{J}_{h}=(\mathcal{I}+h \mathcal{A A})^{-1}$ satisfies the estimates

$$
\left\|J_{h}\right\|_{\mathcal{L}\left(X^{s}\right)} \leq C, \quad\left\|A J_{h}\right\|_{\mathcal{L}\left(X^{s}\right)} \leq C h^{-1}
$$

Proof of Lemma 3.1. As noticed in Section 2, (RK2) implies that the singular points of $(1+z \mathcal{A})^{-1}$ are contained in the set $\Sigma=\left\{z \in \mathbb{C} ;\|\mathcal{A}\|^{-1} \leq|z| \leq\right.$ $\left.\left\|\mathcal{A}^{-1}\right\|,|\arg z| \geq \theta\right\}$. 
Let us define a bounded operator on $X^{s}$ by the integral

$$
\begin{aligned}
J_{h} & =\frac{1}{2 \pi i} \int_{\Gamma}(\mathcal{I}+h \mathcal{A} \lambda)^{-1}(\lambda-A)^{-1} d \lambda \\
& =\frac{1}{2 \pi i} \int_{\Gamma}(\mathcal{I}+\mathcal{A} \lambda)^{-1}\left(\frac{\lambda}{h}-A\right)^{-1} \frac{d \lambda}{h}
\end{aligned}
$$

with the integral contour $\lambda: \lambda=r e^{ \pm i \psi}(0 \leq r<\infty)$, where $\varphi<\psi<\theta$. Since we can change the contour to a bounded one, for example,

$$
\begin{aligned}
& \gamma:\{z ;|z|=\delta,|\arg z| \geq \psi\} \\
& \quad \cup\left\{z ; \delta \leq|z| \leq \delta^{-1}, \arg z= \pm \psi\right\} \cup\left\{z ;|z|=\delta^{-1},|\arg z| \geq \psi\right\},
\end{aligned}
$$

where $0<\delta<\min \left\{\|\mathcal{A}\|^{-1},\left\|\mathcal{A}^{-1}\right\|^{-1}\right\}$, we have:

$$
J_{h}=\frac{1}{2 \pi i} \int_{\gamma}(\mathcal{I}+\mathcal{A} z)^{-1}\left(\frac{z}{h}-A\right)^{-1} \frac{d z}{h} .
$$

Then by a direct calculation it is verified that $(\mathcal{I}+h \mathcal{A} A) \boldsymbol{J}_{h}=\mathcal{I}$. Therefore, $(\mathcal{I}+h \mathcal{A} A)^{-1}$ exists and is given by $\boldsymbol{J}_{h}$.

In addition, since

$$
\left\|J_{h}\right\|_{\mathcal{L}\left(X^{s}\right)} \leq \frac{M}{2 \pi} \int_{\gamma}\left\|(\mathcal{I}+\mathcal{A} z)^{-1}\right\||z|^{-1}|d z|
$$

we observe that $\left\|J_{h}\right\|_{\mathcal{L}\left(X^{s}\right)}$ is uniformly bounded with respect to $h$. In a similar way, we see that $\left\|A J_{h}\right\|_{\mathcal{L}\left(X^{s}\right)} \leq C h^{-1}$.

The operator $R(h A)$ plays, as verified by (3.4), a very important role in describing the approximation solution $U_{n}$. We here notice that $R(h A)$ is a functional calculus of $A$ for the stability function $R(h \lambda)$. Indeed, let $\varepsilon>0$. By $A(\theta)$-stability we have:

$$
\left|(\lambda+1)^{-\varepsilon} R(h \lambda)\right| \leq C(|\lambda|+1)^{-\varepsilon}, \quad \lambda \in \Gamma,
$$

where $\Gamma$ is the integral contour introduced above. Then by (3.5) we observe that

$$
(A+1)^{-\varepsilon} R(h A)=\frac{1}{2 \pi i} \int_{\Gamma}(\lambda+1)^{-\varepsilon} R(h \lambda)(\lambda-A)^{-1} d \lambda .
$$

Therefore, in view of (2.1), we conclude that

$$
\begin{aligned}
R(h A) & =\lim _{\varepsilon \rightarrow 0} \frac{1}{2 \pi i} \int_{\Gamma}(\lambda+1)^{-\varepsilon} R(h \lambda)(\lambda-A)^{-1} d \lambda \\
& =R(\infty)+\frac{1}{2 \pi i} \int_{\Gamma}\{R(h \lambda)-R(\infty)\}(\lambda-A)^{-1} d \lambda
\end{aligned}
$$


Using this fact we show the first property of $R(h A)$.

Proposition 3.2.

$$
\left\|R(h A)^{n}\right\|_{\mathcal{L}(X)} \leq C, \quad n=0,1, \ldots, N+1
$$

Proof. It suffices to consider the case $n \geq 1$. Since

$$
R(h A)^{n}=\lim _{\varepsilon \rightarrow 0} \frac{1}{2 \pi i} \int_{\Gamma}(\lambda+1)^{-\varepsilon} R(h \lambda)^{n}(\lambda-A)^{-1} d \lambda,
$$

we observe that

$$
R(h A)^{n}=R(\infty)^{n}+\frac{1}{2 \pi i} \int_{\Gamma}\left\{R(h \lambda)^{n}-R(\infty)^{n}\right\}(\lambda-A)^{-1} d \lambda .
$$

For each $n$, let us change $\Gamma$ to the following bounded one

$$
\begin{aligned}
\gamma_{n}= & \left\{z ;|z|=\delta^{-1},|\arg z| \geq \psi\right\} \cup\left\{z ; \delta \leq|z| \leq \delta^{-1}, \arg z= \pm \psi\right\} \\
& \cup\{z ; \delta / n \leq|z| \leq \delta, \arg z= \pm \psi\} \cup\{z ;|z|=\delta / n,|\arg z| \geq \psi\} \\
= & \gamma_{n}^{(1)} \cup \gamma_{n}^{(2)} \cup \gamma_{n}^{(3)} \cup \gamma_{n}^{(4)},
\end{aligned}
$$

where $\delta>0$ denotes the number announced in Section 2. Then first of all it follows that

$$
R(h A)^{n}=R(\infty)^{n}+\frac{1}{2 \pi i} \int_{\gamma_{n}} R(z)^{n}\left(\frac{z}{h}-A\right)^{-1} \frac{d z}{h}
$$

Since $|R(\infty)|<1$ is assumed, let us estimate the integrals on the subcontours. By virtue of (2.3) and (2.7), the integrals on $\gamma_{n}^{(1)}$ and $\gamma_{n}^{(2)}$ are easily seen to be estimated by $C \kappa^{n}, 0<\kappa<1$. The integral on $\gamma_{n}^{(3)}$ is estimated by

$$
C \int_{\gamma_{n}^{(3)}} e^{-\nu|z| n}|z|^{-1}|d z| \leq C \int_{\delta / n}^{\delta} e^{-\nu n r} r^{-1} d r \leq C \int_{\delta}^{\infty} e^{-\nu r} r^{-1} d r .
$$

On the other hand, the integral on $\gamma_{n}^{(4)}$ is estimated by

$$
C \int_{\gamma_{n}^{(4)}} e^{\eta|z| n}|z|^{-1}|d z| \leq C \int_{-\psi}^{\psi} e^{\eta} d \rho
$$

Thus the proof is accomplished.

In the proof of this proposition we verified also that the range of $R(h A)^{n}-$ $R(\infty)^{n}$ is contained in the domain of $A$. Moreover, we show the following estimate. 


\section{Proposition 3.3.}

$$
\left\|A\left\{R(h A)^{n}-R(\infty)^{n}\right\}\right\|_{\mathcal{L}(X)} \leq C\{(n+1) h\}^{-1}, \quad 0 \leq n \leq N+1 .
$$

Proof. We already know the formula

$$
A\left\{R(h A)^{n}-R(\infty)^{n}\right\}=\frac{1}{2 \pi i} \int_{\gamma_{n}} R(z)^{n} A\left(\frac{z}{h}-A\right)^{-1} \frac{d z}{h}
$$

Then we repeat the same estimation as above for this integral dividing $\gamma_{n}$ into $\gamma_{n}^{(1)}, \gamma_{n}^{(2)}, \gamma_{n}^{(3)}$ and $\gamma_{n}^{(4)}$. As a result we obtain that

$$
\left\|A\left\{R(h A)^{n}-R(\infty)^{n}\right\}\right\|_{\mathcal{L}(X)} \leq C h^{-1}\left(\kappa^{n}+n^{-1}\right) .
$$

This means that (3.8) holds.

As a corollary of this proposition we obtain very important estimates.

Proposition 3.4.

$$
\begin{aligned}
\left\|A R(h A)^{n} \boldsymbol{b}^{T} \boldsymbol{J}_{h}\right\|_{\mathcal{L}\left(X^{s}, X\right)} \leq C\{(n+1) h\}^{-1}, & 0 \leq n \leq N+1 \\
\left\|A J_{h} e R(h A)^{n}\right\|_{\mathcal{L}\left(X, X^{s}\right)} \leq C\{(n+1) h\}^{-1}, & 0 \leq n \leq N+1
\end{aligned}
$$

Proof. For the proof of (3.9) it suffices to notice that

$$
A R(h A)^{n} \boldsymbol{b}^{T} \boldsymbol{J}_{h}=R(\infty)^{n} \boldsymbol{b}^{T} A \boldsymbol{J}_{h}+A\left\{R(h A)^{n}-R(\infty)^{n}\right\} \boldsymbol{b}^{T} \boldsymbol{J}_{h}
$$

and that $n|R(\infty)|^{n} \leq C$. The proof of (3.10) is similar.

We now prove the first main result of this section.

Theorem 3.5. Assume the conditions (A1), (F1), (I1) and (RK2). Then, the solution $\left\{U_{n}\right\}$ to (3.3) satisfies the estimate

$$
\left\|U_{n}\right\|_{X} \leq C\left(\left\|u_{0}\right\|_{X}+\|f\|_{\mathcal{C}([0, T] ; X)}\right), \quad n=0,1, \ldots, N+1
$$

Moreover, $U_{n} \in \mathcal{D}(A)$ and the estimate

$$
\left\|A U_{n}\right\|_{X} \leq C\left(\left\|A u_{0}\right\|_{X}+\sigma^{-1}\|f\|_{\mathcal{C}^{\sigma}([0, T] ; X)}\right), \quad n=0,1, \ldots, N+1,
$$

is valid. The constants $C \geq 0$ are independent of $u_{0}$ and $f$. 
Proof. Since $U_{n}$ is given by (3.4), the first estimate (3.11) follows immediately from (3.6). To prove (3.12) we write

$$
\begin{aligned}
A U_{n}=R(h A)^{n} A u_{0} & +h \sum_{l=0}^{n-1} A R(h A)^{n-l-1} \boldsymbol{b}^{T} \boldsymbol{J}_{h}\left\{\boldsymbol{f}\left(\boldsymbol{\tau}_{l}\right)-\boldsymbol{e f}\left(t_{n}\right)\right\} \\
& +\left\{1-R(h A)^{n}\right\} f\left(t_{n}\right),
\end{aligned}
$$

here we used a formula

$$
1-R(h A)^{n}=h \sum_{l=0}^{n-1} R(h A)^{n-l-1} \boldsymbol{b}^{T} A J_{h} e .
$$

Then (3.9) is used to obtain that

$$
\left\|A U_{n}\right\|_{X} \leq C\left(\left\|A u_{0}\right\|_{X}+\|f\|_{\mathcal{C}}\right)+C\|f\|_{\mathcal{C}^{\sigma}} h \sum_{l=0}^{n-1}\{(n-l) h\}^{\sigma-1}
$$

The estimate (3.12) is then shown by $h \sum_{l=0}^{n-1}\{(n-l) h\}^{\sigma-1} \leq C(n h)^{\sigma} \int_{0}^{1}(1-$ $x)^{\sigma-1} d x$ (cf. Lemma A.2 in Appendix).

We now proceed to estimate the error $E_{n}=U_{n}-u\left(t_{n}\right)$. For this purpose we assume that (3.1) admits a sufficiently smooth solution $u$.

Let us define $e_{n}, 0 \leq n \leq N$, and $\boldsymbol{d}_{n}, 0 \leq n \leq N$ by the following formulas

$$
\left\{\begin{array}{l}
e_{n}=u\left(t_{n+1}\right)-u\left(t_{n}\right)-h \boldsymbol{b}^{T} \boldsymbol{u}^{\prime}\left(\boldsymbol{\tau}_{n}\right) \\
\boldsymbol{d}_{n}=\boldsymbol{u}\left(\boldsymbol{\tau}_{n}\right)-\boldsymbol{e u}\left(t_{n}\right)-h \mathcal{A} \boldsymbol{u}^{\prime}\left(\boldsymbol{\tau}_{n}\right)
\end{array}\right.
$$

respectively, where $\boldsymbol{u}\left(\boldsymbol{\tau}_{n}\right)=\left[u\left(t_{n}+c_{1} h\right), \ldots, u\left(t_{n}+c_{s} h\right)\right]^{T}, \boldsymbol{u}^{\prime}\left(\boldsymbol{\tau}_{n}\right)=\left[u^{\prime}\left(t_{n}+\right.\right.$ $\left.\left.c_{1} h\right), \ldots, u^{\prime}\left(t_{n}+c_{s} h\right)\right]^{T}$ and $\tau_{n}=\left(t_{n} \mathcal{I}+h \mathcal{C}\right) e$. We observe that

$$
\left\{\begin{aligned}
u\left(t_{n+1}\right) & =u\left(t_{n}\right)+h \boldsymbol{b}^{T}\left\{-A \boldsymbol{u}\left(\boldsymbol{\tau}_{n}\right)+\boldsymbol{f}\left(\boldsymbol{\tau}_{n}\right)\right\}+e_{n} \\
\boldsymbol{u}\left(\boldsymbol{\tau}_{n}\right) & =\boldsymbol{e} u\left(t_{n}\right)+h \mathcal{A}\left\{-\boldsymbol{A u}\left(\boldsymbol{\tau}_{n}\right)+\boldsymbol{f}\left(\boldsymbol{\tau}_{n}\right)\right\}+\boldsymbol{d}_{n}
\end{aligned}\right.
$$

In view of $(3.3)$ it then follows that

$$
\left\{\begin{aligned}
E_{n+1} & =E_{n}-h \boldsymbol{b}^{T} A \boldsymbol{D}_{n}-e_{n}, \quad n=0,1, \ldots, N \\
\boldsymbol{D}_{n} & =\boldsymbol{e} E_{n}-h \mathcal{A} A \boldsymbol{D}_{n}-\boldsymbol{d}_{n} \\
E_{0} & =0
\end{aligned}\right.
$$

with $\boldsymbol{D}_{n}=\boldsymbol{V}_{n}-\boldsymbol{u}\left(\boldsymbol{\tau}_{n}\right)$. Hence, we have

$$
E_{n+1}=R(h A) E_{n}+h \boldsymbol{b}^{T} A \boldsymbol{J}_{h} \boldsymbol{d}_{n}-e_{n}
$$


Therefore $E_{n}$ is represented by the formula

$$
E_{n}=\sum_{l=0}^{n-1} R(h A)^{n-l-1}\left\{h \boldsymbol{b}^{T} A \boldsymbol{J}_{h} \boldsymbol{d}_{l}-e_{l}\right\}
$$

for every $n=0,1, \ldots, N+1$.

On the other hand, we can obtain integral representaions of $e_{n}$ and $\boldsymbol{d}_{n}$ by utilizing the Taylor expansion

$$
\begin{aligned}
\varphi(h)=\varphi(0)+\frac{h}{1 !} \varphi^{\prime}(0)+\frac{h^{2}}{2 !} \varphi^{\prime \prime} & (0)+\cdots \\
& +\frac{h^{m}}{m !} \varphi^{(m)}(0)+\int_{0}^{h} \frac{(h-t)^{m}}{m !} \varphi^{(m+1)}(t) d t
\end{aligned}
$$

repeatedly. Indeed, apply this expansion to $u\left(t_{n}+t\right)$ with $m=p+1$ and to each function $u^{\prime}\left(t_{n}+c_{i} t\right)$ which is the $i$-th component of $\boldsymbol{u}^{\prime}\left(\boldsymbol{\tau}_{n}\right)$ with $m=p$. Then we have:

$$
\begin{aligned}
e_{n}= & \sum_{k=1}^{p} h^{k}\left(\frac{1}{k !}-\frac{1}{(k-1) !} \boldsymbol{b}^{T} \mathcal{C}^{k-1} \boldsymbol{e}\right) u^{(k)}\left(t_{n}\right) \\
& +\int_{0}^{h}\left(\frac{(h-t)^{p}}{p !} u^{(p+1)}\left(t_{n}+t\right)-\frac{h(h-t)^{p-1}}{(p-1) !} \boldsymbol{b}^{T} \mathcal{C}^{p} \boldsymbol{u}^{(p+1)}\left(\left(t_{n} \mathcal{I}+t \mathcal{C}\right) \boldsymbol{e}\right)\right) d t
\end{aligned}
$$

where $\boldsymbol{u}^{(p+1)}\left(\left(t_{n} \mathcal{I}+t \mathcal{C}\right) \boldsymbol{e}\right)=\left[u^{(p+1)}\left(t_{n}+c_{1} t\right), \ldots, u^{(p+1)}\left(t_{n}+c_{s} t\right)\right]^{T}$. Recalling that the quadratic order is bigger than $p$ (see (1.4)), we obtain from (1.6) that

$$
e_{n}=\int_{0}^{h}\left(\frac{(h-t)^{p}}{p !} u^{(p+1)}\left(t_{n}+t\right)-\frac{h(h-t)^{p-1}}{(p-1) !} \boldsymbol{b}^{T} \mathcal{C}^{p} \boldsymbol{u}^{(p+1)}\left(\left(t_{n} \mathcal{I}+t \mathcal{C}\right) e\right)\right) d t
$$

In a similar way we also obtain the following formula

$$
\begin{aligned}
\boldsymbol{d}_{n}= & \sum_{m=1}^{q} h^{m}\left(\frac{1}{m !} \mathcal{C}^{m} \boldsymbol{e}-\frac{1}{(m-1) !} \mathcal{A C}^{m-1} \boldsymbol{e}\right) u^{(m)}\left(t_{n}\right) \\
& +\int_{0}^{h}\left(\frac{(h-t)^{q}}{q !} \mathcal{C}^{q+1}-\frac{h(h-t)^{q-1}}{(q-1) !} \mathcal{A} \mathcal{C}^{q}\right) \boldsymbol{u}^{(q+1)}\left(\left(t_{n} \mathcal{I}+t \mathcal{C}\right) \boldsymbol{e}\right) d t \\
= & \int_{0}^{h}\left(\frac{(h-t)^{q}}{q !} \mathcal{C}^{q+1}-\frac{h(h-t)^{q-1}}{(q-1) !} \mathcal{A} \mathcal{C}^{q}\right) \boldsymbol{u}^{(q+1)}\left(\left(t_{n} \mathcal{I}+t \mathcal{C}\right) \boldsymbol{e}\right) d t
\end{aligned}
$$

Here we used the relation (1.5).

We now establish the error estimates. 
TheOREM 3.6. Let the conditions (A1), (F1), (I1) and (RK1-2) be satisfied. If $u$ is a solution such that $u \in \mathcal{C}^{p+1}([0, T] ; X) \cap \mathcal{C}^{q+1}([0, T] ; \mathcal{D}(A))$, then the error $E_{n}$ is estimated by

$$
\left\|E_{n}\right\|_{X} \leq C\left(h^{p} \int_{0}^{T}\left\|u^{(p+1)}(t)\right\|_{X} d t+h^{q+1} \int_{0}^{T}\left\|A u^{(q+1)}(t)\right\|_{X} d t\right)
$$

for every $n=0,1, \ldots, N+1$. Moreover, if $u$ satisfies $u \in \mathcal{C}^{p+1}([0, T] ; \mathcal{D}(A))$, then

$$
\left\|A E_{n}\right\|_{X} \leq C\left(h^{p} \int_{0}^{T}\left\|A u^{(p+1)}(t)\right\|_{X} d t+\omega^{-1} h^{q+1}\left\|A u^{(q+1)}\right\|_{\mathcal{C}^{\omega}([0, T] ; X)}\right)
$$

for every $n=0,1, \ldots, N+1$ with any $0<\omega<1$.

$\dot{P}_{\text {Roof. }}$ Consider the case where $u \in \mathcal{C}^{p+1}([0, T] ; X) \cap \mathcal{C}^{q+1}([0, T] ; \mathcal{D}(A))$. From (3.16) we immediately observe that

$$
\left\|e_{n}\right\|_{X} \leq C h^{p} \int_{0}^{h}\left\|u^{(p+1)}\left(t_{n}+t\right)\right\|_{X} d t \leq C h^{p} \int_{t_{n}}^{t_{n+1}}\left\|u^{(p+1)}(t)\right\|_{X} d t .
$$

Similarly from (3.17) it follows that

$$
\left\|A d_{n}\right\|_{X} \leq C h^{q} \int_{0}^{h}\left\|A u^{(q+1)}\left(t_{n}+t\right)\right\|_{X} d t \leq C h^{q} \int_{t_{n}}^{t_{n+1}}\left\|A u^{(q+1)}(t)\right\|_{X} d t .
$$

Therefore from the formula (3.15) we verify (3.18).

Consider next the case where $u \in \mathcal{C}^{p+1}([0, T] ; \mathcal{D}(A))$. As before we have:

$$
\left\|A e_{n}\right\|_{X} \leq C h^{p} \int_{t_{n}}^{t_{n+1}}\left\|A u^{(p+1)}(t)\right\|_{X} d t
$$

On the other hand, the vectors $A d_{n}$ satisfy

$$
\begin{gathered}
\left\|A\left(\boldsymbol{d}_{n}-\boldsymbol{d}_{m}\right)\right\|_{X^{s}} \leq C h^{q} \int_{0}^{h}\left\|A\left\{\boldsymbol{u}^{(q+1)}\left(\left(t_{n} \mathcal{I}+t \mathcal{C}\right) \boldsymbol{e}\right)-\boldsymbol{u}^{(q+1)}\left(\left(t_{m} \mathcal{I}+t \mathcal{C}\right) \boldsymbol{e}\right)\right\}\right\|_{X^{s}} d t \\
\leq C h^{q+1}\{(n-m) h\}^{\omega}\left\|A u^{(q+1)}\right\|_{\mathcal{C}^{\omega}}, \\
\left\|A\left(\boldsymbol{d}_{n}-\boldsymbol{e} d_{n}\right)\right\|_{X^{s}} \leq C h^{q} \int_{0}^{h}\left\|A\left\{\boldsymbol{u}^{(q+1)}\left(\left(t_{n} \mathcal{I}+t \mathcal{C}\right) \boldsymbol{e}\right)-\boldsymbol{e} u^{(q+1)}\left(t_{n}\right)\right\}\right\|_{X^{s}} d t \\
\leq C \omega^{-1} h^{q+1}\left\|A u^{(q+1)}\right\|_{\mathcal{C}^{\omega}}
\end{gathered}
$$

with

$$
d_{n}=\int_{0}^{h}\left(\frac{(h-t)^{q}}{q !} \mathcal{C}^{q+1}-\frac{h(h-t)^{q-1}}{(q-1) !} \mathcal{A} \mathcal{C}^{q}\right) e u^{(q+1)}\left(t_{n}\right) d t
$$


This then shows that by the same argument as in the proof Theorem 3.5 the estimate

$$
\left\|h \sum_{l=0}^{n-1} A R(h A)^{n-l-1} \boldsymbol{b}^{T} \boldsymbol{J}_{h} A \boldsymbol{d}_{l}\right\|_{X} \leq C \omega^{-1} h^{q+1}\left\|A u^{(q+1)}\right\|_{\mathcal{C}^{\omega}}
$$

is true for every $0 \leq n \leq N$. Hence (3.19) is also verified from (3.15).

REMARK 3.1. It is known that, if $f(t)$ is more regular and if $f(t)$ and $u_{0}$ satisfy some compatibility conditions, then the solution $u$ to (3.1) actually possesses the regularity required in Theorem 3.6. For details, see Appendix.

REMARK 3.2. If the solution $u$ possesses the regularity

$$
u \in \bigcap_{k=0}^{p} \mathcal{C}^{k+1}\left([0, T] ; \mathcal{D}\left(A^{p-k}\right)\right)
$$

then we can show better error estimates

$$
\begin{gathered}
\left\|E_{n}\right\|_{X} \leq C h^{p} \sum_{k=0}^{p}\left\|A^{p-k} u^{(k+1)}\right\|_{L^{1}((0, T) ; X)}, \\
\left\|A E_{n}\right\|_{X} \leq C h^{p-1} \sum_{k=0}^{p-1}\left\|A^{p-k} u^{(k+1)}\right\|_{L^{1}((0, T) ; X)}
\end{gathered}
$$

for every $n=0,1, \ldots, N+1$.

This estimate is in fact obtained from the same expansions of $e_{n}$ and $h \boldsymbol{b}^{T} A \boldsymbol{J}_{h} \boldsymbol{d}_{l}$ as above with the aid of another order condition that states

$$
\boldsymbol{b}^{T} \mathcal{A}^{k} \mathcal{C}^{m} \boldsymbol{e}=\frac{1}{(m+1)(m+2) \cdots(m+k+1)}, \quad k \geq 0, m \geq 0, k+m+1 \leq p .
$$

We are able to verify that

$$
\begin{gathered}
\left\|e_{n+1}\right\|_{X} \leq C h^{p+1}\left\|u^{(p+1)}\right\|_{\mathcal{C}([0, T] ; X)} \\
\left\|h \boldsymbol{b}^{T} A J_{h} \boldsymbol{d}_{n}\right\|_{X} \leq C h^{p+1} \sum_{k=0}^{p-1}\left\|A^{p-k} u^{(k+1)}\right\|_{\mathcal{C}([0, T] ; X)}
\end{gathered}
$$

and

$$
\begin{gathered}
\left\|A e_{n+1}\right\| \leq C h^{p}\left\|A u^{(p)}\right\|_{\mathcal{C}([0, T] ; X)} \\
\left\|h \boldsymbol{b}^{T} A J_{h} A \boldsymbol{d}_{n}\right\|_{X} \leq C h^{p} \sum_{k=0}^{p-1}\left\|A^{p-k} u^{(k+1)}\right\|_{\mathcal{C}([0, T] ; X)}
\end{gathered}
$$

for every $n=0,1, \ldots, N+1$. 


\section{Non autonomous linear equations}

We consider an approximate solution for the Cauchy problem of a non autonomous linear evolution equation

$$
\left\{\begin{array}{l}
\frac{d u}{d t}+A(t) u=f(t), \quad 0<t \leq T, \\
u(0)=u_{0}
\end{array}\right.
$$

in a Banach space $X$. Here, $-A(t), 0 \leq t \leq T$, are the infinitesimal generators of analytic semigroups on $X$ with domains $\mathcal{D}(A(t))$ independent of $t$.

Assume the following.

(A2) The resolvent sets $\rho(A(t)), 0 \leq t \leq T$, contain a sector $\mathbb{C} \backslash \overline{S_{\varphi}}$, and the resolvents $(\lambda-A(t))^{-1}$ satisfy the same estimate as (3.2) uniformly in $t$, that is,

$$
\left\|(\lambda-A(t))^{-1}\right\|_{\mathcal{L}(X)} \leq \frac{M}{|\lambda|+1}, \quad \lambda \notin \overline{S_{\varphi}}, 0 \leq t \leq T .
$$

(A3) The domains $\mathcal{D}(A(t)) \equiv \mathcal{D}$ are constant, and, for some exponent $0<\mu \leq 1$, $A(\cdot)$ satisfies the Hölder condition

$$
\left\|\{A(t)-A(s)\} A(s)^{-1}\right\|_{\mathcal{L}(X)} \leq L|t-s|^{\mu}, \quad 0 \leq t, s \leq T .
$$

(F2) For a number $\sigma>0, f \in \mathcal{C}^{\sigma}([0, T] ; X)$.

(I2) $u_{0} \in \mathcal{D}(A(0))$.

For the scheme we assume (RK1) and (RK2).

Each $A(t)$ satisfies the assumptions stated in Section 3. Therefore, we can use the results established there.

Let $0<h \leq T$ and let $N \geq 0$ be an integer such that $(N+1) h \leq T$. The R-K scheme for the problem (4.1) is written as

$$
\left\{\begin{aligned}
U_{n+1} & =U_{n}+h \boldsymbol{b}^{T}\left\{-\boldsymbol{A}\left(\boldsymbol{\tau}_{n}\right) \boldsymbol{V}_{n}+\boldsymbol{f}\left(\boldsymbol{\tau}_{n}\right)\right\}, \quad n=0,1, \ldots, N, \\
\boldsymbol{V}_{n} & =\boldsymbol{e} U_{n}+h \mathcal{A}\left\{-\boldsymbol{A}\left(\boldsymbol{\tau}_{n}\right) \boldsymbol{V}_{n}+\boldsymbol{f}\left(\boldsymbol{\tau}_{n}\right)\right\}, \\
U_{0} & =u_{0},
\end{aligned}\right.
$$

where $\boldsymbol{A}\left(\boldsymbol{\tau}_{n}\right)=\operatorname{diag}\left[A\left(t_{n}+c_{1} h\right), \ldots, A\left(t_{n}+c_{s} h\right)\right], \boldsymbol{f}\left(\boldsymbol{\tau}_{n}\right)=\left[f\left(t_{n}+c_{1} h\right), \ldots, f\left(t_{n}+\right.\right.$ $\left.\left.c_{s} h\right)\right]^{T}$ and $\tau_{n}=\left(t_{n} \mathcal{I}+h \mathcal{C}\right) e$.

Let $h$ be sufficiently small. Since Lemma 4.1 below is verified, we can write this scheme in the form

$$
\left\{\begin{aligned}
U_{n+1} & =U_{n}+h \boldsymbol{b}^{T}\left\{-\boldsymbol{A}\left(\boldsymbol{\tau}_{n}\right) \boldsymbol{V}_{n}+\boldsymbol{f}\left(\boldsymbol{\tau}_{n}\right)\right\}, \quad n=0,1, \ldots, N, \\
\boldsymbol{V}_{n} & =\boldsymbol{J}_{h}\left(\boldsymbol{\tau}_{n}\right)\left\{\boldsymbol{e} U_{n}+h \mathcal{A} \boldsymbol{f}\left(\boldsymbol{\tau}_{n}\right)\right\}, \\
U_{0} & =u_{0}
\end{aligned}\right.
$$


where $\boldsymbol{J}_{h}\left(\boldsymbol{\tau}_{n}\right)=\left(\mathcal{I}+h \mathcal{A A}\left(\boldsymbol{\tau}_{n}\right)\right)^{-1} \in \mathcal{L}\left(X^{s}\right)$. Moreover, we can obtain the following formula which gives the approximation solution

$$
U_{n}=\Phi_{h}(n, 0) u_{0}+h \sum_{l=0}^{n-1} \Phi_{h}(n, l+1) \boldsymbol{b}^{T} \mathcal{A}^{-1} \boldsymbol{J}_{h}\left(\boldsymbol{\tau}_{l}\right) \mathcal{A} \boldsymbol{f}\left(\boldsymbol{\tau}_{l}\right), \quad 0 \leq n \leq N+1,
$$

by introducing the linear operators

$$
\Phi_{h}(n, m)= \begin{cases}1, & n=m \\ \left\{1-h \boldsymbol{b}^{T} \boldsymbol{A}\left(\boldsymbol{\tau}_{n-1}\right) \boldsymbol{J}_{h}\left(\boldsymbol{\tau}_{n-1}\right) e\right\} & 0 \leq m<n \leq N+1 \\ \cdots\left\{1-h \boldsymbol{b}^{T} \boldsymbol{A}\left(\boldsymbol{\tau}_{m}\right) \boldsymbol{J}_{h}\left(\boldsymbol{\tau}_{m}\right) \boldsymbol{e}\right\}, & \end{cases}
$$

As will be verified below by several propositions, this family of operators enjoys many properties which are quite analogous to the fundamental solution for the original problem (4.1) and in fact plays an analogous role for the approximation problem. In this sense we call $\Phi_{h}(n, m), 0 \leq m \leq n \leq N+1$, the fundamental solution for (4.2).

Lemma 4.1. There exists a number $h_{0}>0$ such that $\boldsymbol{J}_{h}\left(\boldsymbol{\tau}_{n}\right)=$ $\left(\mathcal{I}+h \mathcal{A} \boldsymbol{A}\left(\boldsymbol{\tau}_{n}\right)\right)^{-1}, 0 \leq n \leq N$, exist as bounded operators provided that $0<h \leq h_{0}$. In addition, the estimates

$$
\left\|\boldsymbol{J}_{h}\left(\boldsymbol{\tau}_{n}\right)\right\|_{\mathcal{L}\left(X^{s}\right)} \leq C, \quad\left\|\boldsymbol{A}\left(\boldsymbol{\tau}_{n}\right) \boldsymbol{J}_{h}\left(\boldsymbol{\tau}_{n}\right)\right\|_{\mathcal{L}\left(X^{s}\right)} \leq C h^{-1}, \quad 0 \leq n \leq N
$$

hold for $0<h \leq h_{0}$.

Proof of Lemma 4.1. We notice that

$$
\mathcal{I}+h \mathcal{A A}\left(\boldsymbol{\tau}_{n}\right)=\left[\mathcal{I}+h \mathcal{A}\left\{\boldsymbol{A}\left(\boldsymbol{\tau}_{n}\right)-A\left(t_{n}\right)\right\} A\left(t_{n}\right)^{-1} A\left(t_{n}\right) \boldsymbol{J}_{h}\left(t_{n}\right)\right]\left(\mathcal{I}+h \mathcal{A} A\left(t_{n}\right)\right)
$$

Then (A3) together with Lemma 3.1 yields that there exists some $h_{0}>0$ such that

$$
\left\|h \mathcal{A}\left\{\boldsymbol{A}\left(\tau_{n}\right)-A\left(t_{n}\right)\right\} A\left(t_{n}\right)^{-1} A\left(t_{n}\right) \boldsymbol{J}_{h}\left(t_{n}\right)\right\|_{\mathcal{L}\left(X^{s}\right)} \leq \frac{1}{2}
$$

for $0<h \leq h_{0}$. Therefore, it follows that

$$
\boldsymbol{J}_{h}\left(\boldsymbol{\tau}_{n}\right)=\boldsymbol{J}_{h}\left(t_{n}\right)\left[\mathcal{I}+h \mathcal{A}\left\{\boldsymbol{A}\left(\boldsymbol{\tau}_{n}\right)-A\left(t_{n}\right)\right\} \boldsymbol{J}_{h}\left(t_{n}\right)\right]^{-1}
$$

The first estimate also follows immediately from this formula. The second one is verified by operating $\boldsymbol{A}\left(\boldsymbol{\tau}_{n}\right)$ to this formula in view of $\left\|\boldsymbol{A}\left(\tau_{n}\right) A\left(t_{n}\right)^{-1}\right\|_{\mathcal{L}\left(X^{s}\right)} \leq C$.

Throughout this section we assume that $0<h \leq h_{0}$. 
Let us verify various properties of $\Phi_{h}(n, m)$. By definition, we observe that

$$
\Phi_{h}(n, l)=\Phi_{h}(n, m) \Phi_{h}(m, l), \quad 0 \leq l \leq m \leq n \leq N+1 .
$$

Proposition 4.2.

$$
\left\|\Phi_{h}(n, m)\right\|_{\mathcal{L}(X)} \leq C, \quad 0 \leq m \leq n \leq N+1 .
$$

Proof. Consider the difference

$$
\begin{aligned}
& \Phi_{h}(n, m)-R\left(h A\left(t_{m}\right)\right)^{n-m} \\
&=\sum_{l=m}^{n-1} \Phi_{h}(n, l+1)\left\{\Phi_{h}(l+1, l)\right.\left.-R\left(h A\left(t_{m}\right)\right)\right\} R\left(h A\left(t_{m}\right)\right)^{l-m} \\
&=h \sum_{l=m}^{n-1} \Phi_{h}(n, l+1) \boldsymbol{b}^{T} \boldsymbol{A}\left(\boldsymbol{\tau}_{l}\right) \boldsymbol{J}_{h}\left(\boldsymbol{\tau}_{l}\right)\left\{\boldsymbol{A}\left(\boldsymbol{\tau}_{l}\right)^{-1}-A\left(t_{m}\right)^{-1}\right\} \\
& \times A\left(t_{m}\right) \boldsymbol{J}_{h}\left(t_{m}\right) \boldsymbol{e} R\left(h A\left(t_{m}\right)\right)^{l-m} \\
&=h \sum_{l=m}^{n-1} \Phi_{h}(n, l+1) \boldsymbol{b}^{T} \mathcal{A}^{-1} \boldsymbol{J}_{h}\left(\boldsymbol{\tau}_{l}\right) \mathcal{A}\left\{\mathcal{I}-\boldsymbol{A}\left(\boldsymbol{\tau}_{l}\right) A\left(t_{m}\right)^{-1}\right\} \\
& \times A\left(t_{m}\right) \boldsymbol{J}_{h}\left(t_{m}\right) \boldsymbol{e} R\left(h A\left(t_{m}\right)\right)^{l-m} .
\end{aligned}
$$

Then, by (3.10) and (A3) we obtain the following inequality

$$
\left\|\Phi_{h}(n, m)\right\|_{\mathcal{L}(X)} \leq C+C h \sum_{l=m}^{n-1}\left\|\Phi_{h}(n, l+1)\right\|_{\mathcal{L}(X)}((l-m+1) h)^{\mu-1}
$$

of Volterra type. (4.4) is then proved as a direct consequence of this inequality. Indeed, use Proposition A.1 in Appendix.

Proposition 4.3.

$$
\left\|A\left(t_{n}\right) \Phi_{h}(n, m) A\left(t_{m}\right)^{-1}\right\|_{\mathcal{L}(X)} \leq C, \quad 0 \leq m \leq n \leq N+1
$$

Proof. In this case consider the difference

$$
\begin{aligned}
\Phi_{h}(n, m)-R\left(h A\left(t_{n}\right)\right)^{n-m} \\
=-\sum_{l=m}^{n-1} R\left(h A\left(t_{n}\right)\right)^{n-l-1}\left\{R\left(h A\left(t_{n}\right)\right)-\Phi_{h}(l+1, l)\right\} \Phi_{h}(l, m) \\
=h \sum_{l=m}^{n-1} R\left(h A\left(t_{n}\right)\right)^{n-l-1} \boldsymbol{b}^{T} \mathcal{A}^{-1} \boldsymbol{J}_{h}\left(t_{n}\right) \mathcal{A} \\
\quad \times\left\{A\left(t_{n}\right) \boldsymbol{A}\left(\boldsymbol{\tau}_{l}\right)^{-1}-\mathcal{I}\right\} \boldsymbol{A}\left(\boldsymbol{\tau}_{l}\right) \boldsymbol{J}_{h}\left(\boldsymbol{\tau}_{l}\right) \boldsymbol{e} \Phi_{h}(l, m) .
\end{aligned}
$$


From this we verify that

$$
\begin{aligned}
& A\left(t_{n}\right) \Phi_{h}(n, m) A\left(t_{m}\right)^{-1} \\
& =R\left(h A\left(t_{n}\right)\right)^{n-m} A\left(t_{n}\right) A\left(t_{m}\right)^{-1} \\
& \quad+h \sum_{l=m}^{n-1} A\left(t_{n}\right) R\left(h A\left(t_{n}\right)\right)^{n-l-1} \boldsymbol{b}^{T} \mathcal{A}^{-1} \boldsymbol{J}_{h}\left(t_{n}\right) \mathcal{A}\left\{A\left(t_{n}\right) \boldsymbol{A}\left(\boldsymbol{\tau}_{l}\right)^{-1}-\mathcal{I}\right\} \\
& \quad \times \mathcal{A}^{-1} \boldsymbol{J}_{h}\left(\boldsymbol{\tau}_{l}\right) \mathcal{A} \boldsymbol{A}\left(\boldsymbol{\tau}_{l}\right) A\left(t_{l}\right)^{-1} \boldsymbol{e} A\left(t_{l}\right) \Phi_{h}(l, m) A\left(t_{m}\right)^{-1}
\end{aligned}
$$

Furthermore, by (3.9) and (A3) the following inequality

$$
\begin{aligned}
& \left\|A\left(t_{n}\right) \Phi_{h}(n, m) A\left(t_{m}\right)^{-1}\right\|_{\mathcal{L}(X)} \\
& \quad \leq C+C h \sum_{l=m}^{n-1}((n-l) h)^{\mu-1}\left\|A\left(t_{l}\right) \Phi_{h}(l, m) A\left(t_{m}\right)^{-1}\right\|_{\mathcal{L}(X)}
\end{aligned}
$$

is obtained. By virtue of Proposition A.1 we conclude (4.5).

Proposition 4.4. The range of $\Phi_{h}(n, m)-R\left(h A\left(t_{n}\right)\right)^{n-m}$ is contained in the domain of $A\left(t_{n}\right)$. In addition, the estimate

$$
\left\|A\left(t_{n}\right)\left\{\Phi_{h}(n, m)-R\left(h A\left(t_{n}\right)\right)^{n-m}\right\}\right\|_{\mathcal{L}(X)} \leq C((n-m+1) h)^{\mu-1}
$$

holds for $0 \leq m \leq n \leq N+1$.

Proof. The first assertion is verified immediately from (4.6). Hence the family of bounded operators

$$
\Psi_{h}(n, m)=A\left(t_{n}\right)\left\{\Phi_{h}(n, m)-R\left(h A\left(t_{n}\right)\right)^{n-m}\right\}, \quad 0 \leq m \leq n \leq N+1
$$

can be defined. From $(4.6), \Psi(n, m)$ is shown to satisfy that

$$
\begin{aligned}
\Psi_{h}(n, m)=Q_{h} & (n, m)+h \sum_{l=m}^{n-1} A\left(t_{n}\right) R\left(h A\left(t_{n}\right)\right)^{n-l-1} \boldsymbol{b}^{T} \mathcal{A}^{-1} \boldsymbol{J}_{h}\left(t_{n}\right) \mathcal{A} \\
& \times\left\{A\left(t_{n}\right) \boldsymbol{A}\left(\boldsymbol{\tau}_{l}\right)^{-1}-\mathcal{I}\right\} \mathcal{A}^{-1} \boldsymbol{J}_{h}\left(\boldsymbol{\tau}_{l}\right) \mathcal{A} \boldsymbol{A}\left(\boldsymbol{\tau}_{l}\right) A\left(t_{l}\right)^{-1} e \Psi_{h}(l, m),
\end{aligned}
$$

where

$$
\begin{aligned}
& Q_{h}(n, m)=h \sum_{l=m}^{n-1} A\left(t_{n}\right) R\left(h A\left(t_{n}\right)\right)^{n-l-1} \boldsymbol{b}^{T} \mathcal{A}^{-1} \boldsymbol{J}_{h}\left(t_{n}\right) \mathcal{A} \\
& \times\left\{A\left(t_{n}\right) \boldsymbol{A}\left(\boldsymbol{\tau}_{l}\right)^{-1}-\mathcal{I}\right\} \boldsymbol{A}\left(\boldsymbol{\tau}_{l}\right) \boldsymbol{J}_{h}\left(\boldsymbol{\tau}_{l}\right) \boldsymbol{e} R\left(h A\left(t_{l}\right)\right)^{l-m} .
\end{aligned}
$$


As a consequence the following inequality

(4.8) $\left\|\Psi_{h}(n, m)\right\|_{\mathcal{L}(X)} \leq\left\|Q_{h}(n, m)\right\|_{\mathcal{L}(X)}+C h \sum_{l=m}^{n-1}((n-l) h)^{\mu-1}\left\|\Psi_{h}(l, m)\right\|_{\mathcal{L}(X)}$ is verified.

Let us now estimate the norm of $Q_{h}(n, m)$. We first divide the summands as

$$
Q_{h}(n, m)=\sum_{l=k}^{n-1}+\sum_{l=m}^{k-1}=Q_{h}^{(1)}(n, m)+Q_{h}^{(2)}(n, m)
$$

with $k=[(n+m) / 2]$. Then,

$$
\left\|Q_{h}^{(1)}(n, m)\right\|_{\mathcal{L}(X)} \leq C h \sum_{l=k}^{n-1}\{(n-l) h\}^{\mu-1}\{(l-m+1) h\}^{-1},
$$

here we used that $\left\|\boldsymbol{A}\left(\boldsymbol{\tau}_{l}\right) \boldsymbol{J}_{h}\left(\boldsymbol{\tau}_{l}\right)\left(\mathcal{I}+h \mathcal{A} A\left(t_{l}\right)\right) A\left(t_{l}\right)^{-1}\right\|_{\mathcal{L}(X)} \leq C$. Therefore,

$$
\begin{aligned}
\left\|Q_{h}^{(1)}(n, m)\right\|_{\mathcal{L}(X)} & \leq C\{(k-m+1) h\}^{-1} \int_{k h}^{n h}(n h-x)^{\mu-1} d x \\
& \leq C\{(n-m+1) h\}^{\mu-1}
\end{aligned}
$$

Next $Q_{h}^{(2)}(n, m)$ is written in the form

$$
\begin{aligned}
Q_{h}^{(2)}(n, m)= & h \sum_{l=m}^{k-1} A\left(t_{n}\right) R\left(h A\left(t_{n}\right)\right)^{n-l-1} \boldsymbol{b}^{T} \mathcal{A}^{-1} \boldsymbol{J}_{h}\left(t_{n}\right) \mathcal{A} \\
& \times A\left(t_{n}\right)\left\{\boldsymbol{J}_{h}\left(\boldsymbol{\tau}_{l}\right) \boldsymbol{e} R\left(h A\left(t_{l}\right)\right)^{l-m}-\boldsymbol{J}_{h}\left(t_{m}\right) \boldsymbol{e} R\left(h A\left(t_{m}\right)\right)^{l-m}\right\} \\
+ & h \sum_{l=m}^{k-1} A\left(t_{n}\right) R\left(h A\left(t_{n}\right)\right)^{n-l-1} \boldsymbol{b}^{T} \mathcal{A}^{-1} \boldsymbol{J}_{h}\left(t_{n}\right) \mathcal{A} \\
& \times\left\{A\left(t_{m}\right) \boldsymbol{J}\left(t_{m}\right) \boldsymbol{e} R\left(h A\left(t_{m}\right)\right)^{l-m}-\boldsymbol{A}\left(\boldsymbol{\tau}_{l}\right) \boldsymbol{J}_{h}\left(\boldsymbol{\tau}_{l}\right) \boldsymbol{e} R\left(h A\left(t_{l}\right)\right)^{l-m}\right\} \\
+ & h \sum_{l=m}^{k-1} A\left(t_{n}\right) R\left(h A\left(t_{n}\right)\right)^{n-l-1} \boldsymbol{b}^{T} \mathcal{A}^{-1} \boldsymbol{J}_{h}\left(t_{n}\right) \mathcal{A} \\
& \times\left\{A\left(t_{n}\right)-A\left(t_{m}\right)\right\} \boldsymbol{J}_{h}\left(t_{m}\right) \boldsymbol{e} R\left(h A\left(t_{m}\right)\right)^{l-m} \\
= & Q_{h}^{(3)}(n, m)+Q_{h}^{(4)}(n, m)+Q_{h}^{(5)}(n, m) .
\end{aligned}
$$

To estimte the norms of these operators we here prepare the following two lemmas. 
LEMMA 4.5.

$$
\begin{aligned}
\left\|\boldsymbol{A}\left(\boldsymbol{\tau}_{n}\right)\left\{\boldsymbol{J}_{h}\left(\boldsymbol{\tau}_{n}\right)-\boldsymbol{J}_{h}\left(\boldsymbol{\tau}_{m}\right)\right\} \boldsymbol{A}\left(\boldsymbol{\tau}_{m}\right)^{-1}\right\|_{\mathcal{L}\left(X^{s}\right)} \\
\quad \leq C\{(n-m) h\}^{\mu}, \quad 0 \leq m \leq n \leq N .
\end{aligned}
$$

Proof of Lemma 4.5. We have:

$$
\boldsymbol{J}_{h}\left(\boldsymbol{\tau}_{n}\right)-\boldsymbol{J}_{h}\left(\boldsymbol{\tau}_{m}\right)=h \boldsymbol{J}_{h}\left(\boldsymbol{\tau}_{n}\right) \mathcal{A}\left\{\mathcal{I}-\boldsymbol{A}\left(\boldsymbol{\tau}_{n}\right) \boldsymbol{A}\left(\boldsymbol{\tau}_{m}\right)^{-1}\right\} \mathcal{A}^{-1} \boldsymbol{J}_{h}\left(\boldsymbol{\tau}_{m}\right) \mathcal{A} \boldsymbol{A}\left(\boldsymbol{\tau}_{m}\right) .
$$

Then the result follows easily from Lemma 4.1.

LEMMA 4.6.

$$
\begin{aligned}
\left\|A(t)\left\{R(h A(t))^{n}-R(h A(s))^{n}\right\}\right\|_{\mathcal{L}(X)} \leq C|t-s|^{\mu}((n+1) h)^{-1} & \\
0 & \leq n \leq N, \quad 0 \leq t, \quad s \leq T .
\end{aligned}
$$

Proof of Lemma 4.6. We have to use the formula (3.7) obtained in the proof of Proposition 3.2. We can in fact write that

$$
\begin{aligned}
A(t)\left\{R(h A(t))^{n}-R(h A(s))^{n}\right\}= & \frac{1}{2 \pi i} \int_{\gamma_{n}} R(z)^{n} A(t)\left(\frac{z}{h}-A(t)\right)^{-1} \\
& \times\left\{1-A(t) A(s)^{-1}\right\} A(s)\left(\frac{z}{h}-A(s)\right)^{-1} \frac{d z}{h} .
\end{aligned}
$$

Then the desired estimate is obtained by the same calculation as for (3.8) in Proposition 3.3.

These two lemmas together with Proposition 3.4 yield that

$$
\begin{aligned}
\left\|Q_{h}^{(3)}(n, m)\right\|_{\mathcal{L}(X)} & \leq C h \sum_{l=m}^{k-1}\{(n-l) h\}^{-1}\{(l-m+1) h\}^{\mu-1} \\
& \leq C\{(n-k+1) h\}^{-1} \int_{m h}^{k h}(x-m h)^{\mu-1} d x \\
& \leq C\{(n-m+1) h\}^{\mu-1} .
\end{aligned}
$$

The same estimate is true for $Q_{h}^{(4)}(n, m)$ also.

Finally we verify directly that

$$
\begin{aligned}
& Q_{h}^{(5)}(n, m)= A\left(t_{n}\right) R\left(h A\left(t_{n}\right)\right)^{n-k} \sum_{l=m}^{k-1} R\left(h A\left(t_{n}\right)\right)^{k-l-1} h \boldsymbol{b}^{T} \\
& \times\left\{A\left(t_{n}\right) J_{h}\left(t_{n}\right)-A\left(t_{m}\right) J_{h}\left(t_{m}\right)\right\} e R\left(h A\left(t_{m}\right)\right)^{l-m} \\
&=R\left(h A\left(t_{n}\right)\right)^{n-k} A\left(t_{n}\right)\left\{R\left(h A\left(t_{n}\right)\right)^{k-l}-R\left(h A\left(t_{m}\right)\right)^{k-l}\right\} .
\end{aligned}
$$


Therefore, it follows that

$$
\left\|Q_{h}^{(5)}(n, m)\right\|_{\mathcal{L}(X)} \leq C\{(n-m+1) h\}^{\mu-1} .
$$

In this way we have proved that

$$
\left\|Q_{h}(n, m)\right\|_{\mathcal{L}(X)} \leq C\{(n-m+1) h\}^{\mu-1} .
$$

The proof of Proposition 4.4 is now completed if we apply Proposition A.1 to (4.8).

As a corollary we observe the following results.

\section{Proposition 4.7.}

$$
\begin{aligned}
& \begin{array}{l}
\left\|A\left(t_{n}\right) \Phi_{h}(n, m+1) \boldsymbol{b}^{T} \mathcal{A}^{-1} \boldsymbol{J}_{h}\left(\boldsymbol{\tau}_{m}\right)\right\|_{\mathcal{L}\left(X^{s}, X\right)} \\
\quad \leq C\{(n-m) h\}^{-1}, \quad 0 \leq m<n \leq N+1, \\
\left\|A\left(t_{n}\right) \boldsymbol{J}_{h}\left(t_{n}\right) \boldsymbol{e} \Phi_{h}(n, m)\right\|_{\mathcal{L}\left(X, X^{s}\right)} \\
\leq C\{(n-m+1) h\}^{-1}, \quad 0 \leq m \leq n \leq N+1 .
\end{array}
\end{aligned}
$$

Proof. We have:

$$
\begin{aligned}
& A\left(t_{n}\right) \Phi_{h}(n, m+1) \boldsymbol{b}^{T} \mathcal{A}^{-1} \boldsymbol{J}_{h}\left(\boldsymbol{\tau}_{m}\right) \\
&= A\left(t_{n}\right)\left\{\Phi_{h}(n, m+1)-R\left(h A\left(t_{n}\right)\right)^{n-m-1}\right\} \boldsymbol{b}^{T} \mathcal{A}^{-1} \boldsymbol{J}_{h}\left(\boldsymbol{\tau}_{m}\right) \\
&+A\left(t_{n}\right) R\left(h A\left(t_{n}\right)\right)^{n-m-1} \boldsymbol{b}^{T} \mathcal{A}^{-1} \boldsymbol{J}_{h}\left(t_{n}\right)\left(\mathcal{I}+h \mathcal{A} A\left(t_{n}\right)\right) \boldsymbol{J}_{h}\left(\boldsymbol{\tau}_{m}\right) .
\end{aligned}
$$

Therefore, (4.9) is verified from (3.9) and (4.7).

The proof for (4.10) is similar if we notice $(3.10)$.

Now we prove the main results of this section.

TheOREM 4.8. Assume the conditions (A2-3), (F2), (I2) and (RK2), and let $(N+1) h \leq T$ with sufficiently small $h>0$. Then, the solution $\left\{U_{n}\right\}$ of (4.2) satisfies the estimate

$$
\left\|U_{n}\right\|_{X} \leq C\left(\left\|u_{0}\right\|_{X}+\|f\|_{\mathcal{C}([0, T] ; X)}\right), \quad n=0,1, \ldots, N+1 .
$$

In addition, $U_{n} \in \mathcal{D}\left(A\left(t_{n}\right)\right)$ and the estimate

$$
\left\|A\left(t_{n}\right) U_{n}\right\|_{X} \leq C\left(\left\|A(0) u_{0}\right\|_{X}+\sigma^{-1}\|f\|_{\mathcal{C}^{\sigma}([0, T] ; X)}\right), \quad n=0,1, \ldots, N+1,
$$


is valid. The constants $C>0$ are independent of $u_{0}$ and $f$.

Proof. Since $U_{n}$ is given by (4.3), (4.11) is an immediate consequence of (4.4). To prove (4.12) we write

$$
\begin{aligned}
A\left(t_{n}\right) U_{n}= & A\left(t_{n}\right) \Phi_{h}(n, 0) A(0)^{-1} A(0) u_{0} \\
& +h \sum_{l=0}^{n-1} A\left(t_{n}\right) \Phi_{h}(n, l+1) \boldsymbol{b}^{T} \mathcal{A}^{-1} \boldsymbol{J}_{h}\left(\boldsymbol{\tau}_{l}\right) \mathcal{A} \boldsymbol{f}\left(\boldsymbol{\tau}_{l}\right)=I^{(1)}+I^{(2)}
\end{aligned}
$$

In view of (4.5) the norm of $I^{(1)}$ is estimated by $C\left\|A(0) u_{0}\right\|_{X}$. On the other hand, $I^{(2)}$ is divided into several terms

$$
\begin{aligned}
I^{(2)}= & h \sum_{l=0}^{n-1} A\left(t_{n}\right)\left\{\Phi_{h}(n, l+1)-R\left(h A\left(t_{n}\right)\right)^{n-l-1}\right\} \boldsymbol{b}^{T} \mathcal{A}^{-1} \boldsymbol{J}_{h}\left(\boldsymbol{\tau}_{l}\right) \mathcal{A} \boldsymbol{f}\left(\boldsymbol{\tau}_{l}\right) \\
+ & h \sum_{l=0}^{n-1} A\left(t_{n}\right) R\left(h A\left(t_{n}\right)\right)^{n-l-1} \boldsymbol{b}^{T} \boldsymbol{J}_{h}\left(t_{n}\right) \\
& \times\left\{\mathcal{I}-A\left(t_{n}\right) \boldsymbol{A}\left(\boldsymbol{\tau}_{l}\right)^{-1}\right\} h \boldsymbol{A}\left(\boldsymbol{\tau}_{l}\right) \boldsymbol{J}_{h}\left(\boldsymbol{\tau}_{l}\right) \mathcal{A} \boldsymbol{f}\left(\boldsymbol{\tau}_{l}\right) \\
+ & h \sum_{l=0}^{n-1} A\left(t_{n}\right) R\left(h A\left(t_{n}\right)\right)^{n-l-1} \boldsymbol{b}^{T} \mathcal{A}^{-1} \boldsymbol{J}_{h}\left(t_{n}\right) \mathcal{A}\left\{\boldsymbol{f}\left(\boldsymbol{\tau}_{l}\right)-e f\left(t_{n}\right)\right\} \\
& +\left\{1-R\left(h A\left(t_{n}\right)\right)^{n}\right\} f\left(t_{n}\right) .
\end{aligned}
$$

Then by (4.7) and (3.9) we show that (4.12) is also true.

We next consider the error $E_{n}=U_{n}-u\left(t_{n}\right), n=0, \ldots, N+1$. Let the solution $u$ to (4.1) be smooth, and let $e_{n}$ and $\boldsymbol{d}_{n}$ denote the same elements as in Section 3. Then, instead of (3.14), we see that

$$
\left\{\begin{aligned}
u\left(t_{n+1}\right) & =u\left(t_{n}\right)+h \boldsymbol{b}^{T}\left\{-\boldsymbol{A}\left(\boldsymbol{\tau}_{n}\right) \boldsymbol{u}\left(\boldsymbol{\tau}_{n}\right)+\boldsymbol{f}\left(\boldsymbol{\tau}_{n}\right)\right\}+e_{n}, \\
\boldsymbol{u}\left(\boldsymbol{\tau}_{n}\right) & =\boldsymbol{e} u\left(t_{n}\right)+h \mathcal{A}\left\{-\boldsymbol{A}\left(\boldsymbol{\tau}_{n}\right) \boldsymbol{u}\left(\boldsymbol{\tau}_{n}\right)+\boldsymbol{f}\left(\boldsymbol{\tau}_{n}\right)\right\}+\boldsymbol{d}_{n}
\end{aligned}\right.
$$

for $0 \leq n \leq N$. This together with (4.2) gives that

$$
\left\{\begin{aligned}
E_{n+1} & =E_{n}-h \boldsymbol{b}^{T} \boldsymbol{A}\left(\boldsymbol{\tau}_{n}\right) \boldsymbol{D}_{n}-e_{n}, \quad n=0,1, \ldots, N \\
\boldsymbol{D}_{n} & =\boldsymbol{e} E_{n}-h \mathcal{A} \boldsymbol{A}\left(\boldsymbol{\tau}_{n}\right) \boldsymbol{D}_{n}-\boldsymbol{d}_{n} \\
E_{0} & =0
\end{aligned}\right.
$$

where $D_{n}=V_{n}-\boldsymbol{u}\left(\tau_{n}\right)$. As a result we obtain the representation formula

$$
E_{n}=\sum_{l=0}^{n-1} \Phi_{h}(n, l+1)\left\{h \boldsymbol{b}^{T} \boldsymbol{A}\left(\boldsymbol{\tau}_{l}\right) \boldsymbol{J}_{h}\left(\boldsymbol{\tau}_{l}\right) \boldsymbol{d}_{l}-e_{l}\right\}
$$


for every $0 \leq n \leq N+1$.

We prove the following estimates.

Theorem 4.9. Let the conditions (A2-3), (F2), (I2) and (RK1-2) be satisfied. If the solution $u$ satisfies $u \in \mathcal{C}^{p+1}([0, T] ; X) \cap \mathcal{C}^{q+1}([0, T] ; \mathcal{D})$, then $E_{n}$ is estimated by

$$
\left\|E_{n}\right\|_{X} \leq C\left(h^{p} \int_{0}^{T}\left\|u^{(p+1)}(t)\right\|_{X} d t+h^{q+1} \int_{0}^{T}\left\|A(t) u^{(q+1)}(t)\right\|_{X} d t\right)
$$

for every $0 \leq n \leq N+1$. Moreover if $u \in \mathcal{C}^{p+1}([0, T] ; \mathcal{D})$, then

$$
\begin{aligned}
\left\|A\left(t_{n}\right) E_{n}\right\|_{X} \leq C\left(h^{p} \int_{0}^{T}\right. & \left\|A(t) u^{(p+1)}(t)\right\|_{X} d t \\
& \left.+\omega^{-1} h^{q+1}\left\|A(\cdot) u^{(q+1)}(\cdot)\right\|_{\mathcal{C}^{\omega}([0, T] ; X)}\right)
\end{aligned}
$$

for every $0 \leq n \leq N+1$ with any $0<\omega<1$.

Proof. Consider the case where $u \in \mathcal{C}^{p+1}([0, T] ; X) \cap \mathcal{C}^{q+1}([0, T] ; \mathcal{D})$. We have verified (3.20). On the other hand, from (3.17) it is seen that

$$
\left\|\boldsymbol{A}\left(\boldsymbol{\tau}_{n}\right) \boldsymbol{d}_{n}\right\|_{X^{s}} \leq C h^{q} \int_{t_{n}}^{t_{n+1}}\left\|A(t) u^{(q+1)}(t)\right\|_{X} d t
$$

Therefore (4.14) is verified from (4.13), noting that $\boldsymbol{A}\left(\boldsymbol{\tau}_{n}\right) \boldsymbol{J}_{h}\left(\boldsymbol{\tau}_{n}\right) \boldsymbol{d}_{n}=\mathcal{A}^{-1} \boldsymbol{J}_{h}\left(\boldsymbol{\tau}_{n}\right) \mathcal{A}$ $\times \boldsymbol{A}\left(\boldsymbol{\tau}_{n}\right) \boldsymbol{d}_{n}$.

Consider next the case where $u \in \mathcal{C}^{p+1}([0, T] ; \mathcal{D})$. From $(3.16)$ it is easy to verify that

$$
\left\|A\left(t_{n}\right) e_{n}\right\|_{X} \leq C h^{p} \int_{t_{n}}^{t_{n+1}}\left\|A(t) u^{(p+1)}(t)\right\|_{X} d t
$$

On the other hand, in order to estimate $\boldsymbol{A}\left(\boldsymbol{\tau}_{n}\right) \boldsymbol{d}_{n}$, let us use again $d_{n}$ defined by (3.22). It is not difficult to verify that

$$
\left\|\boldsymbol{A}\left(\boldsymbol{\tau}_{n}\right) \boldsymbol{d}_{n}-e A\left(t_{n}\right) d_{n}\right\|_{X^{s}} \leq C h^{q+1}\left(h^{\mu}\left\|A u^{(q+1)}\right\|_{\mathcal{C}}+h^{\omega}\left\|A u^{(q+1)}\right\|_{\mathcal{C}^{\omega}}\right)
$$

and

$$
\begin{aligned}
& \left\|A\left(t_{n}\right) d_{n}-A\left(t_{m}\right) d_{m}\right\|_{X} \\
& \quad \leq C h^{q+1}\left(\{(n-m) h\}^{\mu}\left\|A u^{(q+1)}\right\|_{\mathcal{C}}+\{(n-m) h\}^{\omega}\left\|A u^{(q+1)}\right\|_{\mathcal{C}^{\omega}}\right) .
\end{aligned}
$$


Then we can repeat the same argument as in the proof of Theorem 4.8, replacing $\boldsymbol{f}\left(\boldsymbol{\tau}_{n}\right)$ and $f\left(t_{n}\right)$ with $\boldsymbol{A}\left(\boldsymbol{\tau}_{n}\right) \boldsymbol{d}_{n}$ and $A\left(t_{n}\right) d_{n}$, respectively. Consequently we obtain that

$$
\left\|h \sum_{l=0}^{n-1} A\left(t_{n}\right) \Phi_{h}(n, l+1) \boldsymbol{b}^{T} \mathcal{A}^{-1} \boldsymbol{J}_{h}\left(\boldsymbol{\tau}_{l}\right) \mathcal{A} \boldsymbol{A}\left(\boldsymbol{\tau}_{l}\right) \boldsymbol{d}_{l}\right\|_{X} \leq C h^{q+1} \omega^{-1}\left\|A u^{(q+1)}\right\|_{\mathcal{C}^{\omega}}
$$

Hence (4.15) is verified from (4.13).

Remark 4.1. We remark that Theorems 4.8 and 4.9 and all other related results have been derived essentially from the following four conditions:

$$
\begin{aligned}
& \left\|\left\{A\left(t_{n}\right)-A\left(t_{m}\right)\right\} A\left(t_{m}\right)^{-1}\right\|_{\mathcal{L}(X)} \leq L\{(n-m) h\}^{\mu}, \quad 0 \leq m \leq n \leq N+1 \\
& \left\|\left\{\boldsymbol{A}\left(\boldsymbol{\tau}_{n}\right)-A\left(t_{n}\right)\right\} A\left(t_{n}\right)^{-1}\right\|_{\mathcal{L}\left(X, X^{s}\right)} \leq C h^{\mu}, \quad 0 \leq n \leq N+1 \\
& \left\|f\left(t_{n}\right)-f\left(t_{m}\right)\right\|_{X} \leq C\{(n-m) h\}^{\sigma}, \quad 0 \leq m \leq n \leq N+1 \\
& \left\|\boldsymbol{f}\left(\tau_{n}\right)-e f\left(t_{n}\right)\right\|_{X} \leq C h^{\sigma}, \quad 0 \leq n \leq N+1
\end{aligned}
$$

REMARK 4.2. Under suitable regularity conditions on $A(t)$ and $f(t)$ together with some compatibility conditions, the regularity of the solution $u$ required in Theorem 4.9 is actually verified. For details, see Appendix.

REMARK 4.3. Let us assume that

$$
A(\cdot) A(0)^{-1} \in \mathcal{C}^{p}([0, T] ; \mathcal{L}(X))
$$

and that the solution $u$ has the regularity

$$
A(\cdot)^{p-k} u^{(k+1)}(\cdot) \in \mathcal{C}([0, T] ; X)
$$

for $0 \leq k \leq p$. Then, as for Remark 3.2, we can show that

$$
\begin{aligned}
\left\|E_{n}\right\|_{X} & \leq C h^{p} \sum_{k=0}^{p}\left\|A(\cdot)^{p-k} u^{(k+1)}(\cdot)\right\|_{L^{1}((0, T) ; X)} \\
\left\|A\left(t_{n}\right) E_{n}\right\|_{X} & \leq C h^{p-1} \sum_{k=0}^{p-1}\left\|A(\cdot)^{p-k} u^{(k+1)}(\cdot)\right\|_{L^{1}((0, T) ; X)}
\end{aligned}
$$

for every $0 \leq n \leq N+1$. 


\section{Quasilinear equations}

In this section we apply the R-K method to the Cauchy problem of a quasilinear evolution equation

$$
\left\{\begin{array}{l}
\frac{d u}{d t}+A(u) u=f(u), \quad 0<t \leq T \\
u(0)=u_{0}
\end{array}\right.
$$

in a Banach space $X$. Here, $-A(u)$ are the infinitesimal generators of analytic semigroups on $X$ and are defined for elements $u$ in an open ball $K=\{u \in Z ; \| u-$ $\left.u_{0} \|_{Z}<R\right\}$ of $Z, Z(\subset X)$ being another Banach space embedded continuously in $X$. The domains $\mathcal{D}(A(u))$ of $A(u)$ are independent of $u \in K$.

Here we assume the following conditions.

(A4) The resolvent sets $\rho(A(u)), u \in K$, contain a sector $\mathbb{C} \backslash \overline{S_{\varphi}}$, and the resolvents $(\lambda-A(u))^{-1}$ satisfy the same estimate

$$
\left\|(\lambda-A(u))^{-1}\right\|_{\mathcal{L}(X)} \leq \frac{M_{A}}{|\lambda|+1}, \quad \lambda \notin \overline{S_{\varphi}}, u \in K,
$$

as (3.2) with some constant $M_{A}$ independent of $u$.

(A5) The domains $\mathcal{D}(A(u)) \equiv \mathcal{D}$ are constant, and $A(u)$ satisfies the Lipschitz condition

$$
\left\|\{A(u)-A(v)\} A(v)^{-1}\right\|_{\mathcal{L}(X)} \leq L_{A}\|u-v\|_{Z}, \quad u, v \in K .
$$

(Sp) For some $0<\alpha<1, \mathcal{D}\left(A\left(u_{0}\right)^{\alpha}\right)(\subset X)$ is continuously embedded in $Z$.

(F3) $f(u)$ is a given function defined for $u \in K$ with values in $X$ and satisfies the Lipschitz condition

$$
\|f(u)-f(v)\|_{X} \leq L_{f}\|u-v\|_{Z}, \quad u, v \in K,
$$

with some constant $L_{f}$.

(I3) The initial value $u_{0}$ is in $\mathcal{D}\left(A\left(u_{0}\right)\right)$.

Under these assumptions it is already known, see Appendix, that (5.1) has a unique local solution

$$
u \in \mathcal{C}^{\eta}([0, S] ; Z) \cap \mathcal{C}^{1}([0, S] ; X), \quad \text { where } \quad 0<\eta<1-\alpha,
$$

for sufficiently small $(T \geq) S>0$. Under these assumptions we shall also construct an R-K solution $\left\{U_{0}, U_{1}, \ldots, U_{N+1}\right\}$ which approximates the known solution $u(t)$.

In order to estimate the error, however, we shall need to assume more regularity for $A(u)$ and $f(u)$. 
(A6) For each $w \in \mathcal{D}$, the function $A(u) w$ of $u \in K$ with values in $X$ is Fréchet differentiable, and the derivative denoted by $A^{\prime}(u)[\cdot, w] \in \mathcal{L}(Z, X)$ satisfies the Lipschitz condition

$$
\left\|A^{\prime}(u)[\cdot, w]-A^{\prime}(v)[\cdot, w]\right\|_{\mathcal{L}(Z, X)} \leq L_{A^{\prime}}\|u-v\|_{Z}\|w\|_{\mathcal{D}}, u, v \in K, w \in \mathcal{D}
$$

with some constant $L_{A^{\prime}}$ independent of $w$, where $\mathcal{D}$ is considered as a Banach space with the graph norm $\left\|A\left(u_{0}\right) \cdot\right\|_{X}$.

(F4) $f(u)$ is Fréchet differentiable, and the derivative $f^{\prime}(u)[\cdot] \in \mathcal{L}(Z, X)$ satisfies the Lipschitz condition

$$
\left\|f^{\prime}(u)[\cdot]-f^{\prime}(v)[\cdot]\right\|_{\mathcal{L}(Z, X)} \leq L_{f^{\prime}}\|u-v\|_{Z}, \quad u, v \in K
$$

with some constant $L_{f^{\prime}}$.

Formally the R-K scheme for obtaining $\left\{U_{0}, U_{1}, \ldots, U_{N+1}\right\}$ is written as follows.

$$
\left\{\begin{aligned}
U_{n+1} & =U_{n}+h \boldsymbol{b}^{T}\left\{-\boldsymbol{A}\left(\boldsymbol{V}_{n}\right) \boldsymbol{V}_{n}+\boldsymbol{f}\left(\boldsymbol{V}_{n}\right)\right\}, \quad n=0,1, \ldots, N, \\
\boldsymbol{V}_{n} & =\boldsymbol{e} U_{n}+h \mathcal{A}\left\{-\boldsymbol{A}\left(\boldsymbol{V}_{n}\right) \boldsymbol{V}_{n}+\boldsymbol{f}\left(\boldsymbol{V}_{n}\right)\right\}, \\
U_{0} & =u_{0},
\end{aligned}\right.
$$

where $\boldsymbol{A}(\boldsymbol{V})=\operatorname{diag}\left[A\left(V_{1}\right), \ldots, A\left(V_{s}\right)\right]$ and $\boldsymbol{f}(\boldsymbol{V})=\left[f\left(V_{1}\right), \ldots, f\left(V_{s}\right)\right]^{T}$ for $\boldsymbol{V}=$ $\left[V_{1}, \ldots, V_{s}\right]^{T} \in K^{s}$.

However no solution is obtained immediately, because the second equation in (5.2) is not linear. So we have to apply Banach's fixed point theorem.

Take numbers $S$ and $h$ so that $0<S \leq T$ and $0<h<S$, and let $N \geq 0$ denote an integer such that $(N+1) h \leq S$. We consider the product space of $Z$

$$
\begin{aligned}
& \mathcal{X}=\mathcal{X}_{h}(S)=\{\mathcal{U}= {\left[\boldsymbol{U}, \boldsymbol{V}_{0}, \ldots, \boldsymbol{V}_{N}\right] ; \boldsymbol{U}=\left[U_{0}, \ldots, U_{N+1}\right] \in Z^{N+2}, } \\
&\left.\boldsymbol{V}_{n}=\left[V_{n, 1}, \ldots, V_{n, s}\right] \in Z^{s} \text { for } 0 \leq n \leq N\right\}=Z^{N+2} \times\left(Z^{s}\right)^{N+1}
\end{aligned}
$$

equipped with the usual product norm. And we define a subset of $\mathcal{X}$ by

$$
\begin{aligned}
\mathcal{K}= & \mathcal{K}_{h}(S)=\left\{\mathcal{U}=\left[\boldsymbol{U}, \boldsymbol{V}_{0}, \ldots, \boldsymbol{V}_{N}\right] \in \mathcal{X} ; U_{0}=u_{0}\right. \\
& \left\|U_{n}-U_{m}\right\|_{Z} \leq(|n-m| h)^{\eta} \text { for } 0 \leq n, m \leq N+1, \\
& \left.\left\|\boldsymbol{V}_{n}-\boldsymbol{e} u_{0}\right\|_{Z^{s}} \leq \frac{R}{2} \text { and }\left\|\boldsymbol{V}_{n}-\boldsymbol{e} U_{n}\right\|_{Z^{s}} \leq h^{\eta} \text { for } 0 \leq n \leq N\right\}
\end{aligned}
$$

where $\eta$ denotes a fixed exponent such that $0<\eta<1-\alpha$. It is easy to observe that $\mathcal{K}$ is a closed subset of $\mathcal{X}$. 
For each element $\mathcal{W}=\left[\boldsymbol{W}, \boldsymbol{Y}_{0}, \ldots, \boldsymbol{Y}_{N}\right] \in \mathcal{K}$, consider the linear equation

$$
\left\{\begin{aligned}
U_{n+1} & =U_{n}+h \boldsymbol{b}^{T}\left\{-\boldsymbol{A}\left(\boldsymbol{Y}_{n}\right) \boldsymbol{V}_{n}+\boldsymbol{f}\left(\boldsymbol{Y}_{n}\right)\right\}, \quad n=0,1, \ldots, N \\
\boldsymbol{V}_{n} & =e U_{n}+h \mathcal{A}\left\{-\boldsymbol{A}\left(\boldsymbol{Y}_{n}\right) \boldsymbol{V}_{n}+\boldsymbol{f}\left(\boldsymbol{Y}_{n}\right)\right\}, \\
U_{0} & =u_{0}
\end{aligned}\right.
$$

with the unknown elements $U_{n} \in X$ and $V_{n} \in \mathcal{D}^{s}$.

As verified below by Lemma 5.1 , if $h$ is sufficiently small, then $\boldsymbol{J}_{h}\left(\boldsymbol{Y}_{n}\right)=$ $\left(\mathcal{I}+h \mathcal{A A}\left(\boldsymbol{Y}_{n}\right)\right)^{-1}$ are bounded operators from $X^{s}$ into $\mathcal{D}^{s}$ for all components $\boldsymbol{Y}_{n}$ in $\mathcal{W}$. Therefore the second equation in (5.3) is solved as $\boldsymbol{V}_{n}=\boldsymbol{J}_{h}\left(\boldsymbol{Y}_{n}\right)\left\{\boldsymbol{e} U_{n}+\boldsymbol{f}\left(\boldsymbol{Y}_{n}\right)\right\}$. This then means as in Section 4 that a unique solution to (5.3) exists which can be written in the form

$$
\left\{\begin{array}{l}
U_{n}=\Phi_{\mathcal{W}}(n, 0) u_{0}+h \sum_{l=0}^{n-1} \Phi_{\mathcal{W}}(n, l+1) \boldsymbol{b}^{T} \mathcal{A}^{-1} \boldsymbol{J}_{h}\left(\boldsymbol{Y}_{l}\right) \mathcal{A} \boldsymbol{f}\left(\boldsymbol{Y}_{l}\right) \\
\boldsymbol{V}_{n}=\boldsymbol{J}_{h}\left(\boldsymbol{Y}_{n}\right)\left\{\boldsymbol{e} U_{n}+h \mathcal{A} \boldsymbol{f}\left(\boldsymbol{Y}_{n}\right)\right\}, \quad n=0,1, \ldots, N+1
\end{array}\right.
$$

by using the fundamental solution $\Phi_{\mathcal{W}}(n, m), 0 \leq m \leq n \leq N+1$,

$$
\Phi_{\mathcal{W}}(n, m)= \begin{cases}1, & m=n, \\ \left\{1-h \boldsymbol{b}^{T} \boldsymbol{A}\left(\boldsymbol{Y}_{n-1}\right) \boldsymbol{J}_{h}\left(\boldsymbol{Y}_{n-1}\right) \boldsymbol{e}\right\} & 0 \leq m<n \leq N+1, \\ \cdots\left\{1-h \boldsymbol{b}^{T} \boldsymbol{A}\left(\boldsymbol{Y}_{m}\right) \boldsymbol{J}_{h}\left(\boldsymbol{Y}_{m}\right) \boldsymbol{e}\right\}, & \end{cases}
$$

corresponding to the element $\mathcal{W}$.

In this way a mapping $\mathcal{T}: \mathcal{K}_{h}(S) \rightarrow X^{N+2} \times\left(\mathcal{D}^{s}\right)^{N+1}$

$$
\mathcal{T}(\mathcal{W})=\left\{U_{0}, \ldots, U_{N+1}, V_{0}, \ldots, V_{N}\right\}
$$

is defined from (5.3).

Lemma 5.1. Let $h>0$ be sufficiently small. Then $\boldsymbol{J}_{h}\left(\boldsymbol{Y}_{n}\right)=\left(\mathcal{I}+h \mathcal{A A}\left(\boldsymbol{Y}_{n}\right)\right)^{-1}$ exist as bounded operators from $X^{s}$ into $\mathcal{D}^{s}$ for all $0 \leq n \leq N$ and the estimates

$$
\left\|\boldsymbol{J}_{h}\left(\boldsymbol{Y}_{n}\right)\right\|_{\mathcal{L}\left(X^{s}\right)} \leq C, \quad\left\|\boldsymbol{A}\left(\boldsymbol{Y}_{n}\right) \boldsymbol{J}_{h}\left(\boldsymbol{Y}_{n}\right)\right\|_{\mathcal{L}\left(X^{s}\right)} \leq C h^{-1}
$$

hold uniformly in $n$ and $h>0$.

Proof of Lemma 5.1. By Proposition 3.1 similar results are true for the operators $\boldsymbol{J}_{h}\left(W_{n}\right)=\left(\mathcal{I}+h \mathcal{A} A\left(W_{n}\right)\right)^{-1}$, here $W_{n}$ are the components of $\mathcal{W}$. Then we can write at least formally

$$
\boldsymbol{J}_{h}\left(\boldsymbol{Y}_{n}\right)=\boldsymbol{J}_{h}\left(W_{n}\right)\left[\mathcal{I}+h \mathcal{A}\left\{\boldsymbol{A}\left(\boldsymbol{Y}_{n}\right)-A\left(W_{n}\right)\right\} A\left(W_{n}\right)^{-1} A\left(W_{n}\right) \boldsymbol{J}_{h}\left(W_{n}\right)\right]^{-1} .
$$


But this can be verified, in fact, since the condition $\mathcal{W} \in \mathcal{K}_{h}(S)$ together with (A5) implies that

$$
\left\|\left\{\boldsymbol{A}\left(\boldsymbol{Y}_{n}\right)-A\left(W_{n}\right)\right\} A\left(W_{n}\right)^{-1}\right\|_{\mathcal{L}\left(X^{s}\right)} \leq C\left\|\boldsymbol{Y}_{n}-e W_{n}\right\|_{Z} \leq C h^{\eta}
$$

The first estimate is also verified. To prove the second estimate it suffices to note that $\left\|\boldsymbol{A}\left(\boldsymbol{Y}_{n}\right) A\left(W_{n}\right)^{-1}\right\|_{\mathcal{L}\left(X^{s}\right)} \leq C$ follows immediately from (A5).

Let us prove that $\mathcal{T}$ is a contraction mapping of $\mathcal{K}$. We begin with verifying the following proposition.

Proposition 5.2. Let $S$ be sufficiently small, then $\mathcal{T}$ maps the set $\mathcal{K}_{h}(S)$ into itself.

Proof. Let $\mathcal{W}=\left[\boldsymbol{W}, \boldsymbol{Y}_{0}, \ldots, \boldsymbol{Y}_{N}\right] \in \mathcal{K}$ and let $\mathcal{U}=\left[\boldsymbol{U}, \boldsymbol{V}_{0}, \ldots, \boldsymbol{V}_{N}\right]=\mathcal{T} \mathcal{W}$ which is given by (5.4).

We here want to use the results in Section 4. According to Remark 4.1, all the results were established essentially under the conditions (4.16), (4.17), (4.18) and (4.19). If we substitute $\boldsymbol{A}\left(\boldsymbol{Y}_{n}\right), A\left(W_{n}\right), \boldsymbol{f}\left(\boldsymbol{Y}_{n}\right)$ and $f\left(W_{n}\right)$ for $\boldsymbol{A}\left(\boldsymbol{\tau}_{n}\right), A\left(t_{n}\right), \boldsymbol{f}\left(\boldsymbol{\tau}_{n}\right)$ and $f\left(t_{n}\right)$, then the conditions corresponding to (4.16), (4.17), (4.18) and (4.19) are shown to hold with $\mu=\sigma=\eta$. For example, from

$$
\left\|\left\{A\left(W_{n}\right)-A\left(W_{m}\right)\right\} A\left(W_{m}\right)^{-1}\right\|_{\mathcal{L}(X)} \leq L_{A}\left\|W_{n}-W_{m}\right\|_{Z} \leq L_{A}(|n-m| h)^{\eta},
$$

(4.16) is verified. It is similar for the others.

Therefore Theorem 4.8 applied to (5.4) yields that

$$
\left\|A\left(W_{n}\right) U_{n}\right\|_{X} \leq C, \quad 0 \leq n \leq N .
$$

On the other hand, since the semigroup property of $\Phi_{\mathcal{W}}(n, m)$ implies

$$
U_{n}=\Phi_{\mathcal{W}}(n, m) U_{m}+h \sum_{l=m}^{n-1} \Phi_{\mathcal{W}}(n, l+1) \boldsymbol{b}^{T} \mathcal{A}^{-1} J_{h}\left(\boldsymbol{Y}_{l}\right) \mathcal{A} \boldsymbol{f}\left(\boldsymbol{Y}_{l}\right)
$$

for $0 \leq m \leq n \leq N+1$, we have:

$$
\begin{aligned}
U_{n}-U_{m}=\left\{\Phi_{\mathcal{W}}(n, m)\right. & -1\} A\left(W_{m}\right)^{-1} A\left(W_{m}\right) U_{m} \\
& +h \sum_{l=m}^{n-1} \Phi_{\mathcal{W}}(n, l+1) \boldsymbol{b}^{T} \mathcal{A}^{-1} \boldsymbol{J}_{h}\left(\boldsymbol{Y}_{l}\right) \mathcal{A} \boldsymbol{f}\left(\boldsymbol{Y}_{l}\right)
\end{aligned}
$$

Therefore Proposition 4.2 together with Lemma 5.3 below yields that

$$
\left\|U_{n}-U_{m}\right\|_{X} \leq C|n-m| h
$$


Then from (5.5) and (5.6) it follows that

$$
\begin{aligned}
\left\|U_{n}-U_{m}\right\|_{Z} & \leq C\left\|A\left(u_{0}\right)^{\alpha}\left(U_{n}-U_{m}\right)\right\|_{X} \leq C\left\|A\left(u_{0}\right)\left(U_{n}-U_{m}\right)\right\|_{X}^{\alpha}\left\|U_{n}-U_{m}\right\|_{X}^{1-\alpha} \\
& \leq C(|n-m| h)^{1-\alpha} \leq C S^{1-\alpha-\eta}(|n-m| h)^{\eta} .
\end{aligned}
$$

In particular if $m=0$ then $\left\|U_{n}-u_{0}\right\|_{Z} \leq C S^{1-\alpha}$.

We now use the second formula of (5.4). Since

$$
\begin{aligned}
\boldsymbol{V}_{n}-\boldsymbol{e} U_{n} & =\left\{\boldsymbol{J}_{h}\left(\boldsymbol{Y}_{n}\right)-\mathcal{I}\right\} \boldsymbol{e} U_{n}+h \boldsymbol{J}_{h}\left(\boldsymbol{Y}_{n}\right) \mathcal{A} \boldsymbol{f}\left(\boldsymbol{Y}_{n}\right) \\
& =-h \boldsymbol{J}_{h}\left(\boldsymbol{Y}_{n}\right) \mathcal{A A}\left(\boldsymbol{Y}_{n}\right) \boldsymbol{e} U_{n}+h \boldsymbol{J}_{h}\left(\boldsymbol{Y}_{n}\right) \mathcal{A} \boldsymbol{f}\left(\boldsymbol{Y}_{n}\right)
\end{aligned}
$$

we verify in view of Lemma 5.1 that

$$
\left\|\boldsymbol{V}_{n}-e U_{n}\right\|_{X^{s}} \leq C h, \quad\left\|\boldsymbol{A}\left(\boldsymbol{Y}_{n}\right)\left(\boldsymbol{V}_{n}-e U_{n}\right)\right\|_{X^{s}} \leq C .
$$

Then in the same way as above it follows that

$$
\left\|V_{n}-e U_{n}\right\|_{Z^{s}} \leq C h^{1-\alpha} \leq C S^{1-\alpha-\eta} h^{\eta}
$$

Thus if $S$ is sufficiently small, then $\mathcal{U}$ belongs to $\mathcal{K}$.

Lemma 5.3.

$$
\left\|\left\{\Phi_{\mathcal{W}}(n, m)-1\right\} A\left(W_{m}\right)^{-1}\right\|_{\mathcal{L}(X)} \leq C(n-m) h, \quad 0 \leq m \leq n \leq N+1
$$

Proof of Lemma 5.3. The equation verified in the proof of Proposition 4.2 gives in the present case that

$$
\begin{aligned}
&\left\{\Phi_{\mathcal{W}}(n, m)-R\left(h A\left(W_{m}\right)\right)^{n-m}\right\} A\left(W_{m}\right)^{-1} \\
&=h \sum_{l=m}^{n-1} \Phi_{\mathcal{W}}(n, l+1) \boldsymbol{b}^{T} \mathcal{A}^{-1} \boldsymbol{J}_{h}\left(\boldsymbol{Y}_{l}\right) \mathcal{A}\left\{\mathcal{I}-\boldsymbol{A}\left(\boldsymbol{Y}_{l}\right) A\left(W_{m}\right)^{-1}\right\} \\
& \times \boldsymbol{J}_{h}\left(W_{m}\right) \boldsymbol{e} R\left(h A\left(W_{m}\right)\right)^{l-m}
\end{aligned}
$$

From this it follows that

$$
\left\|\left\{\Phi_{\mathcal{W}}(n, m)-R\left(h A\left(W_{m}\right)\right)^{n-m}\right\} A\left(W_{m}\right)^{-1}\right\|_{\mathcal{L}(X)} \leq C(n-m) h .
$$

Similarly, it is obtained from (3.13) that

$$
\left\|\left\{R\left(h A\left(W_{m}\right)\right)^{n-m}-1\right\} A\left(W_{m}\right)^{-1}\right\|_{\mathcal{L}(X)} \leq C(n-m) h .
$$

Hence we complete the proof of lemma. 
Proposition 5.4. Let $S$ be sufficiently small. Then, $\mathcal{T}$ is a contraction mapping with respect to the norm $\|\cdot\|_{\mathcal{X}_{h}(S)}$.

Proof. Let $\mathcal{W}, \mathcal{W}^{\prime} \in \mathcal{K}_{h}(S)$ and $\mathcal{U}=\mathcal{T} \mathcal{W}, \mathcal{U}^{\prime}=\mathcal{T} \mathcal{W}^{\prime}$. Then we have:

$$
\begin{aligned}
U_{n}-U_{n}^{\prime}= & \left\{\Phi_{\mathcal{W}}(n, 0)-\Phi_{\mathcal{W}^{\prime}}(n, 0)\right\} u_{0} \\
& +h \sum_{l=0}^{n-1}\left\{\Phi_{\mathcal{W}}(n, l+1)-\Phi_{\mathcal{W}^{\prime}}(n, l+1)\right\} \boldsymbol{b}^{T} \mathcal{A}^{-1} \boldsymbol{J}_{h}\left(\boldsymbol{Y}_{l}^{\prime}\right) \mathcal{A} \boldsymbol{f}\left(\boldsymbol{Y}_{l}^{\prime}\right) \\
& +h \sum_{l=0}^{n-1} \Phi_{\mathcal{W}}(n, l+1) \boldsymbol{b}^{T} \mathcal{A}^{-1}\left\{\boldsymbol{J}_{h}\left(\boldsymbol{Y}_{l}\right) \mathcal{A} \boldsymbol{f}\left(\boldsymbol{Y}_{l}\right)-\boldsymbol{J}_{h}\left(\boldsymbol{Y}_{l}^{\prime}\right) \mathcal{A} \boldsymbol{f}\left(\boldsymbol{Y}_{l}^{\prime}\right)\right\} \\
= & I_{1}+I_{2}+I_{3} .
\end{aligned}
$$

Take a number $\theta$ such that $\alpha<\theta<1$. Since

$$
\begin{aligned}
I_{1}=h & \sum_{l=0}^{n-1} \Phi_{\mathcal{W}}(n, l+1) \boldsymbol{b}^{T} \mathcal{A}^{-1} \boldsymbol{J}_{h}\left(\boldsymbol{Y}_{l}\right) \mathcal{A}\left\{\mathcal{I}-\boldsymbol{A}\left(\boldsymbol{Y}_{l}\right) \boldsymbol{A}\left(\boldsymbol{Y}_{l}^{\prime}\right)^{-1}\right\} \\
& \times \boldsymbol{A}\left(\boldsymbol{Y}_{l}^{\prime}\right) \boldsymbol{J}_{h}\left(\boldsymbol{Y}_{l}^{\prime}\right) A\left(W_{l}^{\prime}\right)^{-1} \boldsymbol{e} A\left(W_{l}^{\prime}\right) \Phi_{\mathcal{W}^{\prime}}(l, 0) A\left(W_{0}^{\prime}\right)^{-1} A\left(u_{0}\right) u_{0}
\end{aligned}
$$

Lemma 5.5 below and Proposition 4.3 yield that

$$
\begin{aligned}
\left\|A\left(W_{n}\right)^{\theta} I_{1}\right\|_{X} & \leq C h \sum_{l=0}^{n-1}\{(n-l) h\}^{-\theta}\left\|\boldsymbol{Y}_{l}-\boldsymbol{Y}_{l}^{\prime}\right\|_{Z^{s}} \\
& \leq C(n h)^{1-\theta} \max _{0 \leq l \leq n-1}\left\|\boldsymbol{Y}_{l}-\boldsymbol{Y}_{l}^{\prime}\right\|_{Z^{s}}
\end{aligned}
$$

By some calculation it is seen that

$$
\begin{aligned}
I_{2}=h & \sum_{j=1}^{n-1} \Phi_{\mathcal{W}}(n, j+1) \boldsymbol{b}^{T} \mathcal{A}^{-1} \boldsymbol{J}_{h}\left(\boldsymbol{Y}_{j}\right) \mathcal{A}\left\{\mathcal{I}-\boldsymbol{A}\left(\boldsymbol{Y}_{j}^{\prime}\right) \boldsymbol{A}\left(\boldsymbol{Y}_{j}\right)^{-1}\right\} \\
& \times \boldsymbol{A}\left(\boldsymbol{Y}_{j}^{\prime}\right) \boldsymbol{J}_{h}\left(\boldsymbol{Y}_{j}^{\prime}\right) A\left(W_{j}\right)^{-1} \boldsymbol{e} \\
& \times h \sum_{l=0}^{j-1} A\left(W_{j}^{\prime}\right) \Phi_{\mathcal{W}^{\prime}}(j, l+1) \boldsymbol{b}^{T} \mathcal{A}^{-1} \boldsymbol{J}_{h}\left(\boldsymbol{Y}_{l}^{\prime}\right) \mathcal{A} \boldsymbol{f}\left(\boldsymbol{Y}_{l}^{\prime}\right)
\end{aligned}
$$

Since $\mathcal{W}^{\prime}=\left[\boldsymbol{W}^{\prime}, \boldsymbol{Y}_{0}^{\prime}, \ldots, \boldsymbol{Y}_{N}^{\prime}\right] \in \mathcal{K}_{h}(S)$ and since $f$ is Lipschitz continuous, we can repeat the same argument as Theorem 4.8 to obtain

$$
\left\|h \sum_{l=0}^{j-1} A\left(W_{j}^{\prime}\right) \Phi_{\mathcal{W}^{\prime}}(j, l+1) \boldsymbol{b}^{T} \mathcal{A}^{-1} J_{h}\left(\boldsymbol{Y}_{l}^{\prime}\right) \mathcal{A} \boldsymbol{f}\left(\boldsymbol{Y}_{l}^{\prime}\right)\right\|_{X} \leq C .
$$


Therefore, Lemma 5.5 below again yields that

$$
\begin{aligned}
\left\|A\left(W_{n}\right)^{\theta} I_{2}\right\|_{X} & \leq C h \sum_{j=0}^{n-1}\{(n-j) h\}^{-\theta}\left\|\boldsymbol{Y}_{j}-\boldsymbol{Y}_{j}^{\prime}\right\|_{Z^{s}} \\
& \leq C(n h)^{1-\theta} \max _{0 \leq j \leq n-1}\left\|\boldsymbol{Y}_{j}-\boldsymbol{Y}_{j}^{\prime}\right\|_{Z^{s}}
\end{aligned}
$$

The estimate for $I_{3}$ follows more directly from Lemma 5.5. Indeed we have:

$$
\begin{aligned}
\left\|A\left(W_{n}\right)^{\theta} I_{3}\right\|_{X} & \leq C h \sum_{l=0}^{n-1}\{(n-l) h\}^{-\theta}\left\|\boldsymbol{Y}_{l}-\boldsymbol{Y}_{l}^{\prime}\right\|_{Z^{s}} \\
& \leq C(n h)^{1-\theta} \max _{0 \leq l \leq n-1}\left\|\boldsymbol{Y}_{l}-\boldsymbol{Y}_{l}^{\prime}\right\|_{Z^{s}}
\end{aligned}
$$

Summing up these estimates we conclude that

$$
\left\|U_{n}-U_{n}^{\prime}\right\|_{Z} \leq C\left\|A\left(W_{n}\right)^{\theta}\left(U_{n}-U_{n}^{\prime}\right)\right\|_{X} \leq C S^{1-\theta} \max _{0 \leq l \leq N}\left\|\boldsymbol{Y}_{l}-\boldsymbol{Y}_{l}^{\prime}\right\|_{Z^{s}} .
$$

Here we used the fact that $\mathcal{D}(A(W))=\mathcal{D}\left(A\left(u_{0}\right)\right)$ implies that $\mathcal{D}\left(A(W)^{\theta}\right) \subset$ $\mathcal{D}\left(A\left(u_{0}\right)^{\alpha}\right)$ for $W \in K$.

Let us now estimate the norm of $\boldsymbol{V}_{n}-\boldsymbol{V}_{n}^{\prime}$. From the second formula of (5.4) we can write that

$$
\begin{aligned}
\boldsymbol{V}_{n}-\boldsymbol{V}_{n}^{\prime}= & \boldsymbol{J}_{h}\left(\boldsymbol{Y}_{n}\right) \boldsymbol{e}\left(U_{n}-U_{n}^{\prime}\right)+\left\{\boldsymbol{J}_{h}\left(\boldsymbol{Y}_{n}\right)-\boldsymbol{J}_{h}\left(\boldsymbol{Y}_{n}^{\prime}\right)\right\} U_{n}^{\prime} \\
& +h\left\{\boldsymbol{J}_{h}\left(\boldsymbol{Y}_{n}\right) \mathcal{A} \boldsymbol{f}\left(\boldsymbol{Y}_{n}\right)-\boldsymbol{J}_{h}\left(\boldsymbol{Y}_{n}^{\prime}\right) \mathcal{A} \boldsymbol{f}\left(\boldsymbol{Y}_{n}^{\prime}\right)\right\}=\boldsymbol{J}_{1}+\boldsymbol{J}_{2}+\boldsymbol{J}_{3}
\end{aligned}
$$

Then, using the estimate obtained above, we verify that

$$
\begin{aligned}
\left\|\boldsymbol{A}\left(\boldsymbol{Y}_{n}\right)^{\theta} \boldsymbol{J}_{1}\right\|_{X^{s}} & \leq C\left\|\boldsymbol{A}\left(\boldsymbol{Y}_{n}\right)^{\theta} A\left(W_{n}\right)^{-\theta^{\prime}} \boldsymbol{e} A\left(W_{n}\right)^{\theta^{\prime}}\left(U_{n}-U_{n}^{\prime}\right)\right\|_{X^{s}} \\
& \leq C S^{1-\theta^{\prime}} \max _{0 \leq l \leq N}\left\|\boldsymbol{Y}_{l}-\boldsymbol{Y}_{l}^{\prime}\right\|_{Z^{s}}
\end{aligned}
$$

with $(\alpha<) \theta<\theta^{\prime}<1$.

For $\boldsymbol{J}_{2}$ we easily observe that

$$
\left\|\boldsymbol{A}\left(\boldsymbol{Y}_{n}\right) \boldsymbol{J}_{2}\right\|_{X^{s}} \leq C\left\|\boldsymbol{Y}_{n}-\boldsymbol{Y}_{n}^{\prime}\right\|_{Z^{s}} .
$$

Hence, $\left\|\boldsymbol{A}\left(\boldsymbol{Y}_{n}\right)^{\theta} J_{2}\right\|_{X^{s}} \leq C S^{1-\alpha} \max _{0 \leq l \leq N}\left\|Y_{l}-Y_{l}^{\prime}\right\|$. The same is true for $J_{3}$. Therefore, it follows that

$$
\left\|V_{n}-V_{n}^{\prime}\right\|_{Z^{s}} \leq C S^{1-\theta^{\prime}} \max _{0 \leq l \leq N}\left\|Y_{l}-Y_{l}^{\prime}\right\|_{Z^{s}} .
$$

We have thus proved that, if $S$ is sufficiently small, then $\mathcal{T}$ is a contraction mapping. 
Lemma 5.5. For any $0<\theta<1$,

$$
\begin{aligned}
\left\|A\left(W_{n}\right)^{\theta} \Phi_{\mathcal{W}}(n, m+1) \boldsymbol{b}^{T} \mathcal{A}^{-1} \boldsymbol{J}_{h}\left(\boldsymbol{Y}_{m}\right)\right\|_{\mathcal{L}\left(X^{s}, X\right)} \\
\quad \leq C_{\theta}\{(n-m) h\}^{-\theta}, \quad 0 \leq m<n \leq N+1,
\end{aligned}
$$

holds with some constant $C_{\theta}$ depending on $\theta$.

Proof of Lemma 5.5. The results which correspond to $\theta=0$ and 1 were already obtained by Propositions 4.2 and 4.7. Hence the desired result follows immediately by the moment inequality

$$
\left\|A\left(W_{n}\right)^{\theta} u\right\| \leq C_{\theta}\left\|A\left(W_{n}\right) u\right\|_{X}^{\theta}\|u\|_{X}^{1-\theta}, \quad u \in \mathcal{D} .
$$

We are now in a position to prove the existence of solution to (5.2).

TheOREM 5.6. Assume the conditions (A4-5), (Sp), (F3), (I3) and (RK2). Let $S>0$ be sufficiently small. Then, in $\mathcal{K}_{h}(S)$ the problem (5.2) has a unique solution $\mathcal{U}=\left[\boldsymbol{U}, \boldsymbol{V}_{0}, \ldots, \boldsymbol{V}_{N}\right]$. In addition, all the components of $\boldsymbol{U}$ are in $\mathcal{D}$ and satisfy the estimates

$$
\left\|A\left(U_{n}\right) U_{n}\right\|_{X} \leq C, \quad n=0,1, \ldots, N+1 .
$$

The constant $C>0$ is independent of $h$ and $n$.

Proof. Obviously $\mathcal{U} \in \mathcal{K}_{h}(S)$ is a solution to (5.2) if and only if $\mathcal{U}$ is a fixed point of the mapping $\mathcal{T}$. Hence the existence and uniqueness is obtained by the fixed point theorem for the contraction mappings. In addition, $\mathcal{U}$ satisfies the formula

$$
U_{n}=\Phi_{\mathcal{U}}(n, 0) u_{0}+h \sum_{l=0}^{n-1} \Phi_{\mathcal{U}}(n, l+1) \boldsymbol{b}^{T} \mathcal{A}^{-1} \boldsymbol{J}_{h}\left(\boldsymbol{V}_{l}\right) \mathcal{A} \boldsymbol{f}\left(\boldsymbol{V}_{l}\right), \quad n=0, \ldots, N+1
$$

The estimate (5.7) follows from (5.5).

Let us proceed to estimate the error $E_{n}=U_{n}-u\left(t_{n}\right), n=0,1, \ldots, N+1$.

Let $u$ be the solution to $(5.1)$ on $[0, S]$. As before $u$ is assumed to be sufficiently smooth. In particular, there exists a constant $\widetilde{C}>0$ such that

$$
\sup _{0 \leq t \leq S}\left\|A\left(u_{0}\right) u(t)\right\|_{X} \leq \widetilde{C}
$$

We again use the elements $e_{n}$ and $\boldsymbol{d}_{n}$ given by (3.16) and (3.17) respectively. In the present case we observe the relation

$$
\left\{\begin{aligned}
u\left(t_{n+1}\right) & =u\left(t_{n}\right)+h \boldsymbol{b}^{T}\left\{-\boldsymbol{A}\left(\boldsymbol{u}\left(\boldsymbol{\tau}_{n}\right)\right) \boldsymbol{u}\left(\boldsymbol{\tau}_{n}\right)+\boldsymbol{f}\left(\boldsymbol{u}\left(\boldsymbol{\tau}_{n}\right)\right)\right\}+e_{n} \\
\boldsymbol{u}\left(\boldsymbol{\tau}_{n}\right) & =\boldsymbol{e} u\left(t_{n}\right)+h \mathcal{A}\left\{-\boldsymbol{A}\left(\boldsymbol{u}\left(\boldsymbol{\tau}_{n}\right)\right) \boldsymbol{u}\left(\boldsymbol{\tau}_{n}\right)+\boldsymbol{f}\left(\boldsymbol{u}\left(\boldsymbol{\tau}_{n}\right)\right)\right\}+\boldsymbol{d}_{n}
\end{aligned}\right.
$$


for each $0 \leq n \leq N$.

Let $\left\{U_{0}, U_{1}, \ldots, U_{N+1}\right\}$ be the solution to (5.2) constructed by Theorem 5.6 on the interval $[0, S]$. As proved, there is a constant $\widetilde{C}>0$ such that

$$
\sup _{0 \leq n \leq N+1}\left\|A\left(u_{0}\right) U_{n}\right\|_{X} \leq \widetilde{C} .
$$

We are concerned with the difference between (5.2) and (5.8). For this we note that

$$
\begin{gathered}
\{A(u)-A(v)\} v=\int_{0}^{1} A^{\prime}(\rho u+(1-\rho) v)[u-v, v] d \rho=B(u, v)(u-v) \\
f(u)-f(v)=\int_{0}^{1} f^{\prime}(\rho u+(1-\rho) v)[u-v] d \rho=F(u, v)(u-v)
\end{gathered}
$$

where $B(u, v)$ and $F(u, v)$ denote the linear operators

$$
\begin{aligned}
& B(u, v) w=\int_{0}^{1} A^{\prime}(\rho u+(1-\rho) v)[w, v] d \rho, \quad u \in K, v \in K \cap \mathcal{D}, w \in Z \\
& F(u, v) w=\int_{0}^{1} f^{\prime}(\rho u+(1-\rho) v)[w] d \rho, \quad u, v \in K, w \in Z
\end{aligned}
$$

respectively. Then the difference is written as

$$
\left\{\begin{aligned}
E_{n+1} & =E_{n}+h \boldsymbol{b}^{T}\left\{-\boldsymbol{A}\left(\boldsymbol{V}_{n}\right)-\boldsymbol{B}\left(\boldsymbol{V}_{n}, \boldsymbol{u}\left(\boldsymbol{\tau}_{n}\right)\right)+\boldsymbol{F}\left(\boldsymbol{V}_{n}, \boldsymbol{u}\left(\boldsymbol{\tau}_{n}\right)\right)\right\} \boldsymbol{D}_{n}-e_{n} \\
\boldsymbol{D}_{n} & =\boldsymbol{e} E_{n}+h \mathcal{A}\left\{-\boldsymbol{A}\left(\boldsymbol{V}_{n}\right)-\boldsymbol{B}\left(\boldsymbol{V}_{n}, \boldsymbol{u}\left(\boldsymbol{\tau}_{n}\right)\right)+\boldsymbol{F}\left(\boldsymbol{V}_{n}, \boldsymbol{u}\left(\boldsymbol{\tau}_{n}\right)\right)\right\} \boldsymbol{D}_{n}-\boldsymbol{d}_{n}
\end{aligned}\right.
$$

for each $0 \leq n \leq N$, where

$$
\begin{aligned}
& \boldsymbol{B}(\boldsymbol{u}, \boldsymbol{v})=\operatorname{diag}\left\{B\left(u_{1}, v_{1}\right), \ldots, B\left(u_{s}, v_{s}\right)\right\} \\
& \boldsymbol{F}(\boldsymbol{u}, \boldsymbol{v})=\operatorname{diag}\left\{F\left(u_{1}, v_{1}\right), \ldots, F\left(u_{s}, v_{s}\right)\right\}
\end{aligned}
$$

for $\boldsymbol{u}=\left[u_{1}, \ldots, u_{s}\right]^{T}$ and $\boldsymbol{v}=\left[v_{1}, \ldots, v_{s}\right]^{T}$. Of course, $E_{0}=0$.

We are then led to introduce the linear operators

$$
\widetilde{A}(u, v)=\beta+A(u)+B(u, v)-F(u, v)
$$

acting in $X$ for $u \in K$ and $v \in \widetilde{K}=\left\{v \in K \cap \mathcal{D} ;\|v\|_{\mathcal{D}} \leq \widetilde{C}\right\}$ (see (5.9)), here $\beta$ denotes a positive constant specified below. Obviously the domains of $\widetilde{A}(u, v)$ coincide with $\mathcal{D}$ for all $(u, v)$.

Moreover, from the assumptions, the following conditions are satisfied. 
Proposition 5.7. Let $\beta$ be sufficiently large. Then, the resolvent sets of $\widetilde{A}(u, v)$ contain the sector $\mathbb{C} \backslash \overline{S_{\varphi}}$, and the resolvents $(\lambda-\widetilde{A}(u, v))^{-1}$ satisfy

$$
\left\|(\lambda-\widetilde{A}(u, v))^{-1}\right\|_{\mathcal{L}(X)} \leq \frac{\widetilde{M}}{|\lambda|+1}, \quad \lambda \notin \overline{S_{\varphi}}
$$

for all $(u, v) \in K \times \widetilde{K}$. In addition, the Lipschitz condition

$$
\begin{array}{r}
\left\|\left\{\widetilde{A}(u, v)-\widetilde{A}\left(u^{\prime}, v^{\prime}\right)\right\} \widetilde{A}\left(u^{\prime}, v^{\prime}\right)^{-1}\right\|_{\mathcal{L}(X)} \leq \widetilde{L}\left\{\left\|u-u^{\prime}\right\|_{z}+\left\|v-v^{\prime}\right\|_{\mathcal{D}}\right\} \\
(u, v),\left(u^{\prime}, v^{\prime}\right) \in K \times \widetilde{K}
\end{array}
$$

holds.

Proof. If $\lambda-\beta \notin \overline{S_{\varphi}}$, then

$$
\lambda-\widetilde{A}(u, v)=\left[1-\{B(u, v)-F(u, v)\}((\lambda-\beta)-A(u))^{-1}\right]((\lambda-\beta)-A(u)) .
$$

From (A6) it is easily verified that

$$
\begin{aligned}
\left\|B(u, v)((\lambda-\beta)-A(u))^{-1}\right\|_{\mathcal{L}(X)} & \leq C\left\|((\lambda-\beta)-A(u))^{-1}\right\|_{\mathcal{L}(X, Z)} \\
& \leq C(|\lambda-\beta|+1)^{\alpha-1}
\end{aligned}
$$

Similarly, from (F4)

$$
\left\|F(u, v)((\lambda-\beta)-A(u))^{-1}\right\|_{\mathcal{L}(X)} \leq C(|\lambda-\beta|+1)^{\alpha-1} .
$$

These then show that, if $\beta$ is sufficiently large, then $\lambda \in \rho(\widetilde{A}(u, v))$ for all $\lambda \notin \overline{S_{\varphi}}$ with

$$
\begin{aligned}
(\lambda-\tilde{A}(u, v))^{-1}=( & (\lambda-\beta)-A(u))^{-1} \\
& \times\left[1-\{B(u, v)-F(u, v)\}((\lambda-\beta)-A(u))^{-1}\right]^{-1} .
\end{aligned}
$$

Hence the first assertion is proved.

The Lipschitz condition is also easily verified from (A5), (A6) and (F4).

For $n=0,1, \ldots, N$, let us set

$$
\begin{gathered}
\widetilde{A}_{n}=\widetilde{A}\left(U_{n}, u\left(t_{n}\right)\right), \\
\widetilde{A}_{n}=\operatorname{diag}\left\{\widetilde{A}\left(V_{n, 1}, u\left(t_{n}+c_{1} h\right)\right), \ldots, \widetilde{A}\left(V_{n, s}, u\left(t_{n}+c_{s} h\right)\right)\right\}
\end{gathered}
$$

where $V_{n}=\left[V_{n, 1}, \ldots, V_{n, s}\right]^{T}$. Then it is easily verified that

$$
\begin{gathered}
\left\|\left\{\tilde{A}_{n}-\widetilde{A}_{m}\right\} \widetilde{A}_{m}^{-1}\right\|_{\mathcal{L}(X)} \leq C\{(n-m) h\}^{\eta}, \quad 0 \leq m \leq n \leq N \\
\left\|\left\{\tilde{A}_{n}-\widetilde{A}_{n} \mathcal{I}\right\} \widetilde{A}_{n}^{-1}\right\|_{\mathcal{L}\left(X^{s}\right)} \leq C h^{\eta}, \quad 0 \leq n \leq N
\end{gathered}
$$


$C$ being dependent on the norm $\|u\|_{\mathcal{C}^{1}([0, T] ; \mathcal{D})}$.

Using these operators, (5.10) is rewritten as

$$
\left\{\begin{aligned}
E_{n+1} & =E_{n}+h \boldsymbol{b}^{T}\left\{-\widetilde{\boldsymbol{A}}_{n}+\beta\right\} \boldsymbol{D}_{n}-e_{n} \\
\boldsymbol{D}_{n} & =\boldsymbol{e} E_{n}+h \mathcal{A}\left\{-\widetilde{\boldsymbol{A}}_{n}+\beta\right\} \boldsymbol{D}_{n}-\boldsymbol{d}_{n} .
\end{aligned}\right.
$$

Here we assume that $h$ is sufficiently small so that

$$
\tilde{\boldsymbol{J}}_{h, n}=\left(\mathcal{I}+h \mathcal{A} \tilde{\boldsymbol{A}}_{n}\right)^{-1}, \quad 0 \leq n \leq N,
$$

exist as bounded operators (cf. Lemma 5.1). Then, since

$$
\left(\mathcal{I}-h \beta \mathcal{A}+h \mathcal{A} \widetilde{\boldsymbol{A}}_{n}\right)^{-1}=\widetilde{\boldsymbol{J}}_{h, n}\left(\mathcal{I}-h \beta \mathcal{A} \widetilde{\boldsymbol{J}}_{h, n}\right)^{-1},
$$

(if necessary we may reduce $h$ again), some calculation yields that

$$
\begin{aligned}
E_{n+1}=\{1 & \left.-h \boldsymbol{b}^{T} \widetilde{\boldsymbol{A}}_{n} \widetilde{\boldsymbol{J}}_{h, n} \boldsymbol{e}\right\} E_{n}+h \boldsymbol{b}^{T} \widetilde{\boldsymbol{A}}_{n} \widetilde{\boldsymbol{J}}_{h, n} \boldsymbol{d}_{n}-e_{n} \\
& +h \beta \boldsymbol{b}^{T} \mathcal{A}^{-1} \widetilde{\boldsymbol{J}}_{h, n} \mathcal{A} \widetilde{\boldsymbol{J}}_{h, n}\left(\mathcal{I}-h \beta \mathcal{A} \widetilde{\boldsymbol{J}}_{h, n}\right)^{-1}\left\{\boldsymbol{e} E_{n}-\boldsymbol{d}_{n}\right\} .
\end{aligned}
$$

As a consequence we obtain the representaion formula

$$
\begin{aligned}
E_{n}=\sum_{l=0}^{n-1} \widetilde{\Phi}_{h}(n, l+1)\left\{h \boldsymbol{b}^{T} \mathcal{A}^{-1} \widetilde{\boldsymbol{J}}_{h, l} \mathcal{A} \widetilde{\boldsymbol{A}}_{l} \boldsymbol{d}_{l}-e_{l}\right\} \\
\quad+\beta h \sum_{l=0}^{n-1} \widetilde{\Phi}_{h}(n, l+1) \boldsymbol{b}^{T} \mathcal{A}^{-1} \widetilde{\boldsymbol{J}}_{h, l} \mathcal{A} \widetilde{\boldsymbol{J}}_{h, l}\left(\mathcal{I}-h \beta \mathcal{A} \widetilde{\boldsymbol{J}}_{h, l}\right)^{-1}\left\{\boldsymbol{e} E_{l}-\boldsymbol{d}_{l}\right\}
\end{aligned}
$$

with the fundamental solution $\widetilde{\Phi}_{h}(n, m)$ with respect to $\widetilde{A}_{n}, 0 \leq n \leq N$.

We will now accomplish the error estimates.

TheOREM 5.8. Assume the conditions (A4-6), (Sp), (F3-4), (I3) and (RK12). Let $S>0$ be sufficiently small. If the solution $u$ to (5.1) satisfies $u \in$ $\mathcal{C}^{p+1}([0, S] ; X) \cap \mathcal{C}^{q+1}([0, S] ; \mathcal{D})$, then the errors $E_{n}, 0 \leq n \leq N+1$, are estimated by

$$
\left\|E_{n}\right\|_{X} \leq C\left(h^{p}\left\|u^{(p+1)}\right\|_{L^{1}([0, S] ; X)}+h^{q+1}\left\|A\left(u_{0}\right) u^{(q+1)}\right\|_{L^{1}([0, S] ; X)}\right),
$$

the constant $C$ being dependent on $\|u\|_{\mathcal{C}^{1}([0, S] ; \mathcal{D})}$. Moreover, if $u$ satisfies $u \in$ $\mathcal{C}^{p+1}([0, S] ; \mathcal{D})$, then

$$
\begin{aligned}
\left\|A\left(u_{0}\right) E_{n}\right\|_{X} \leq C\left(h^{p} \|\right. & A\left(u_{0}\right) u^{(p+1)} \|_{L^{1}([0, S] ; X)} \\
& \left.+\omega^{-1} h^{q+1}\left\|A\left(u_{0}\right) u^{(q+1)}\right\|_{\mathcal{C}^{\omega}([0, S] ; X)}\right)
\end{aligned}
$$


with any $0<\omega<1$, the constant $C$ being dependent on $\|u\|_{\mathcal{C}^{1}([0, S] ; \mathcal{D})}$.

Proof. The operators $\widetilde{A}_{n}$ and $\widetilde{\boldsymbol{A}}_{n}$ satisfy (5.11) and (5.12). Therefore, as remarked in Remark 4.1, the fundamental solution $\widetilde{\Phi}_{h}(n, m)$ enjoys all the properties established in Section 4.

Consider first the case where $u \in \mathcal{C}^{p+1}([0, S] ; X) \cap \mathcal{C}^{q+1}([0, S] ; \mathcal{D})$. Then, by (3.20) and (3.21) we obtain that

$$
\left\|E_{n}\right\|_{X} \leq C \int_{0}^{t_{n}}\left(h^{p}\left\|u^{(p+1)}(t)\right\|_{X}+h^{q+1}\left\|A\left(u_{0}\right) u^{(q+1)}(t)\right\|_{X}\right) d t+C h \sum_{l=0}^{n-1}\left\|E_{l}\right\|_{X} .
$$

Therefore, it follows that

$$
\begin{aligned}
\left\|E_{n}\right\|_{X} & \leq C \sum_{l=0}^{n-1}(C h)^{l} \int_{0}^{t_{n-l}}\left(h^{p}\left\|u^{(p+1)}(t)\right\|_{X}+h^{q+1}\left\|A\left(u_{0}\right) u^{(q+1)}(t)\right\|_{X}\right) d t \\
& \leq C \sum_{l=0}^{n-1}(C h)^{l} \int_{0}^{t_{n}}\left(h^{p}\left\|u^{(p+1)}(t)\right\|_{X}+h^{q+1}\left\|A\left(u_{0}\right) u^{(q+1)}(t)\right\|_{X}\right) d t .
\end{aligned}
$$

Hence we prove (5.13).

Consider next the case where $u \in \mathcal{C}^{p+1}([0, S] ; \mathcal{D})$. By the same argument as for (4.15), we obtain that

$$
\begin{aligned}
\left\|A\left(u_{0}\right) E_{n}\right\|_{X} \leq C & \left(h^{p}\left\|A\left(u_{0}\right) u^{(p+1)}\right\|_{L^{1}\left(\left[0, t_{n}\right] ; X\right)}\right. \\
& \left.+\omega^{-1} h^{q+1}\left\|A\left(u_{0}\right) u^{(q+1)}\right\|_{\mathcal{C}^{\omega}\left(\left[0, t_{n}\right] ; X\right)}\right)+C h \sum_{l=0}^{n-1}\left\|A\left(u_{0}\right) E_{l}\right\|_{X}
\end{aligned}
$$

From this we conclude (5.14).

\section{An application to the chemotactic model}

Let us consider the Keller-Segel equations

$$
\left\{\begin{array}{l}
\frac{\partial u}{\partial t}=\operatorname{div}\{a \nabla u-u b(\rho) \nabla \rho\} \quad \text { in } \Omega \times(0, \infty) \\
\frac{\partial \rho}{\partial t}=d \Delta \rho+f u-g \rho \quad \text { in } \Omega \times(0, \infty) \\
\frac{\partial u}{\partial n}=\frac{\partial \rho}{\partial n}=0 \quad \text { on } \partial \Omega \times(0, \infty), \\
u(x, 0)=u_{0}(x), \rho(x, 0)=\rho_{0}(x) \quad \text { in } \Omega
\end{array}\right.
$$


which was presented as a model for the chemotaxis, see [11]. Here, $\Omega$ is a bounded region in $\mathbb{R}^{2}$ of $\mathcal{C}^{2}$-class, $n(x)$ the outer normal vector at a boundary point $x \in \partial \Omega$. $a, d, f$ and $g$ are positive constants. $b(\rho)$ is a given function satisfying

$$
\left\{\begin{array}{l}
b(\rho) \text { is analytic in } \mathbb{C}^{+}=\{\rho \in \mathbb{C} ; \operatorname{Re} \rho>0\} \\
b(\rho) \geq 0 \text { for } \rho>0
\end{array}\right.
$$

$u_{0}$ and $\rho_{0}$ are the initial functions which are assumed to be

$$
\left\{\begin{array}{l}
u_{0}, \rho_{0} \in H^{2}(\Omega) \\
\frac{\partial u_{0}}{\partial n}=\frac{\partial \rho_{0}}{\partial n}=0 \quad \text { on } \quad \partial \Omega \\
u_{0}(x) \geq 0, \rho_{0}(x) \geq \delta_{0} \quad \text { on } \bar{\Omega}
\end{array}\right.
$$

with a constant $\delta_{0}>0 . u$ and $\rho$ are the unknown functions.

As was done in [34], under these assumptions, we formulate (6.1) as an abstract equation in the product $L^{2}$-space $X=L^{2}(\Omega) \times L^{2}(\Omega)$ of the form $[\cdot]$. Set $Z=$ $H^{1+\varepsilon}(\Omega) \times H^{1+\varepsilon}(\Omega)$ with some fixed $0<\varepsilon<1 / 2$. By $(6.3)$ the initial function $U_{0}=\left[\begin{array}{l}u_{0} \\ \rho_{0}\end{array}\right]$ is in $Z \subset \mathcal{C}(\bar{\Omega})$. Define an open ball

$$
\begin{aligned}
K=\left\{U=\left[\begin{array}{l}
u \\
\rho
\end{array}\right] \in Z ;\right. & \left\|U-U_{0}\right\|_{Z} \\
& \left.=\sqrt{\left\|u-u_{0}\right\|_{H^{1+\varepsilon}(\Omega)}^{2}+\left\|\rho-\rho_{0}\right\|_{H^{1+\varepsilon}(\Omega)}^{2}}<r\right\}
\end{aligned}
$$

in $Z$ with the center $U_{0}$. If $r$ is small, then there exists $\delta>0$ such that

$$
|\operatorname{Im} u(x)|,|\operatorname{Im} \rho(x)|<\delta \text { and } \operatorname{Re} \rho(x) \geq \delta \text { on } \bar{\Omega}
$$

for $U=\left[\begin{array}{l}u \\ \rho\end{array}\right] \in K$.

For $U=\left[\begin{array}{l}u \\ \rho\end{array}\right] \in K$, let us define linear operators $A(U)$ by

$$
\left\{\begin{array}{l}
\mathcal{D}(A(U))=\mathcal{D}=\left\{\tilde{U}=\left[\begin{array}{l}
\widetilde{u} \\
\tilde{\rho}
\end{array}\right] \in H^{2}(\Omega) \times H^{2}(\Omega)\right. \\
\left.\frac{\partial \widetilde{u}}{\partial n}=\frac{\partial \widetilde{\rho}}{\partial n}=0 \text { on } \partial \Omega\right\}, \\
A(U) \widetilde{U}=\left[\begin{array}{c}
-\operatorname{div}\{a \nabla \widetilde{u}-u b(\rho) \nabla \widetilde{\rho}\}+a \tilde{u} \\
-d \triangle \tilde{\rho}+g \widetilde{\rho}
\end{array}\right], \quad \widetilde{U}=\left[\begin{array}{l}
\widetilde{u} \\
\tilde{\rho}
\end{array}\right] .
\end{array}\right.
$$


Then (6.1) is written as

$$
\left\{\begin{array}{l}
\frac{d U}{d t}+A(U) U=F(U), \quad t>0 \\
U(0)=U_{0}
\end{array}\right.
$$

where $F(U)$ denotes the function

$$
F(U)=\left[\begin{array}{l}
a u \\
f u
\end{array}\right], \quad U=\left[\begin{array}{l}
u \\
\rho
\end{array}\right] \in K
$$

Since $A_{1}=-a \triangle+a$ and $A_{2}=-d \triangle+g$ with the domains $\mathcal{D}\left(A_{1}\right)=\mathcal{D}\left(A_{2}\right)=$ $\left\{u \in H^{2}(\Omega) ; \partial u / \partial n=0\right.$ on $\left.\partial \Omega\right\}$ are positive definite self adjoint operators in $L^{2}(\Omega)$, for any $\lambda \in \mathbb{C} \backslash(0, \infty), \lambda-A(U)$ has a bounded inverse

$$
\begin{gathered}
(\lambda-A(U))^{-1} \widetilde{F}=\left[\begin{array}{c}
\left(\lambda-A_{1}\right)^{-1}\left[\tilde{f}-\operatorname{div}\left\{u b(\rho) \nabla\left(\lambda-A_{2}\right)^{-1} \widetilde{g}\right\}\right] \\
\left(\lambda-A_{2}\right)^{-1} \widetilde{g}
\end{array}\right], \\
\widetilde{F}=\left[\begin{array}{c}
\tilde{f} \\
\widetilde{g}
\end{array}\right] \in X .
\end{gathered}
$$

Then, by means of the inequalities

$$
\left\{\begin{array}{l}
\|\operatorname{div}\{b \nabla \rho\}\|_{L^{2}} \leq C_{\varepsilon}\|b\|_{H^{1+\varepsilon}}\|\rho\|_{H^{2}}, \quad b \in H^{1+\varepsilon}(\Omega), \rho \in H^{2}(\Omega), \\
\|u b\|_{H^{1+\varepsilon}} \leq C_{\varepsilon}\|u\|_{H^{1+\varepsilon}}\|b\|_{H^{1+\varepsilon}}, \quad u \in H^{1+\varepsilon}(\Omega), b \in H^{1+\varepsilon}(\Omega),
\end{array}\right.
$$

(A4) is proved with any $0<\varphi<\pi / 2$; for details confer [34]. Also (A5) and (F3) are easily verified. By (6.3), (I3) is obvious.

From (6.4) it is easily seen that the domain of the adjoint operator $A(U)^{*}$ of $A(U)$ coincides with $\mathcal{D}(A(U))=\mathcal{D}$. Then, by [34, Theorem A.1], we have $\mathcal{D}\left(A(U)^{(1+\varepsilon) / 2}\right) \equiv Z, U \in K$, and hence (Sp) is observed.

By a direct calculation it is observed that $A(U) \widetilde{U}, \widetilde{U} \in \mathcal{D}$, is Fréchet differentiable with the derivative

Similarly,

$$
\left\{\begin{aligned}
& \mathcal{D}\left(A^{\prime}(U)\right)=Z \times \mathcal{D} \\
& A^{\prime}(U)[\widetilde{V}, \widetilde{U}]=\left[\begin{array}{c}
\operatorname{div}\left\{\left(b(\rho) \widetilde{v}+u b^{\prime}(\rho) \widetilde{\mu}\right) \nabla \widetilde{\rho}\right\} \\
0
\end{array}\right], \\
& U=\left[\begin{array}{l}
u \\
\rho
\end{array}\right], \quad \tilde{V}=\left[\begin{array}{c}
\widetilde{v} \\
\tilde{\mu}
\end{array}\right], \quad \widetilde{U}=\left[\begin{array}{c}
\widetilde{u} \\
\tilde{\rho}
\end{array}\right] .
\end{aligned}\right.
$$

$$
\left\{\begin{array}{l}
\mathcal{D}\left(F^{\prime}(U)\right)=Z \\
F^{\prime}(U)[\widetilde{V}]=\left[\begin{array}{l}
a \widetilde{v} \\
f \widetilde{v}
\end{array}\right], \quad U=\left[\begin{array}{l}
u \\
\rho
\end{array}\right], \quad \widetilde{V}=\left[\begin{array}{c}
\widetilde{v} \\
\widetilde{\mu}
\end{array}\right] .
\end{array}\right.
$$

Since $b$ and $b^{\prime}$ are smooth in $\mathbb{C}^{+},(\mathrm{A} 6)$ and (F4) are verified. 


\section{Appendix}

\section{A.1. Discrete version for the inequality of Volterra type}

As a discrete version of the inequality of Volterra type, we can prove the following results.

Proposition A.1. Let $h>0$ and let $N \geq 0$ be an integer. Assume that a double sequence $\left\{x_{n, m}\right\}_{0 \leq m \leq n \leq N+1}$ satisfies the inequality

$$
x_{n, m} \leq a\{(n-m+1) h\}^{\alpha-1}+h \sum_{l=m}^{n-1} b\{(n-l) h\}^{\beta-1} x_{l, m}
$$

for all $0 \leq m \leq n \leq N+1$, where $a, b$ are positive constants and $0<\alpha, \beta \leq 1$ are some exponents. Then, $x_{n, m}$ is estimated by

$$
x_{n, m} \leq C\{(n-m+1) h\}^{\alpha-1}, \quad 0 \leq m \leq n \leq N+1
$$

where the constant $C$ is determined by $a, b, \alpha, \beta$ and $(N+1) h$ only.

Proof. By induction we shall prove that

$$
\begin{aligned}
x_{n, m} \leq \sum_{j=0}^{k} a b^{j} & \frac{\Gamma(\alpha) \Gamma(\beta)^{j}}{\Gamma(\alpha+j \beta)}\{(n-m+1) h\}^{\alpha+j \beta-1} \\
& +h \sum_{l=m}^{n-k-1} b^{k+1} \frac{\Gamma(\beta)^{k+1}}{\Gamma((k+1) \beta)}\{(n-l) h\}^{(k+1) \beta-1} x_{l, m}
\end{aligned}
$$

holds for every $0 \leq k \leq n-m$, where $\Gamma(x)$ denotes the gamma function. When $k=0$, this is nothing more than (A.1). Hence, assume that (A.2) holds for some $k \geq 0$. Then, replacing $x_{l, m}, m \leq l \leq n-k-1$, by the terms on the right hand side of (A.1) applied to themselves, we obtain that

$$
\begin{aligned}
x_{n, m} \leq & \sum_{j=0}^{k} a b^{j} \frac{\Gamma(\alpha) \Gamma(\beta)^{j}}{\Gamma(\alpha+j \beta)}\{(n-m+1) h\}^{\alpha+j \beta-1} \\
& +h \sum_{l=m}^{n-k-1} a b^{k+1} \frac{\Gamma(\beta)^{k+1}}{\Gamma((k+1) \beta)}\{(n-l) h\}^{(k+1) \beta-1}\{(l-m+1) h\}^{\alpha-1} \\
& +h \sum_{l^{\prime}=m}^{n-k-2} \sum_{l=l^{\prime}}^{n-k-2} b^{k+2} \frac{\Gamma(\beta)^{k+1}}{\Gamma((k+1) \beta)}\{(n-1-l) h\}^{(k+1) \beta-1} \\
& \times\left\{\left(l-l^{\prime}+1\right) h\right\}^{\beta-1} x_{l^{\prime}, m}
\end{aligned}
$$


here the change of the order of summation is used for $\sum_{l=m}^{n-k-1} \sum_{l^{\prime}=m}^{l-1}$.

Now we notice the following lemma.

Lemma A.2. Let $p>0$ and $0<q<1$. Then,

$$
h \sum_{l=m}^{n-1}\{(n-l) h\}^{p-1}\{(l-m+1) h\}^{q-1} \leq B(p, q)\{(n-m+1) h\}^{p+q-1}
$$

for $0 \leq m \leq n \leq N+1$, where $B(p, q)$ denotes the beta function.

Proof of Lemma A.2. Consider first the case when $0<p<1$. The function $f(x)=((n+1) h-x)^{p-1}(x-m h)^{q-1}$ takes its minimum at some point $x_{0}$ and let $l_{0}$ be the integer such that $l_{0} h \leq x_{0} \leq\left(l_{0}+1\right) h$. Then, since $f(x)$ is decreasing (resp. increasing) in $\left(m h, l_{0} h\right]$ (resp. $\left.\left[\left(l_{0}+1\right) h,(n+1) h\right)\right)$, it is seen that

$$
\begin{aligned}
h \sum_{l=m}^{n-1}\{(n & -l) h\}^{p-1}\{(l-m+1) h\}^{q-1} \\
& \leq\left(\int_{m h}^{l_{0} h}+\int_{\left(l_{0}+1\right) h}^{(n+1) h}\right) f(x) d x \leq \int_{m h}^{(n+1) h} f(x) d x \\
& =B(p, q)\{(n-m+1) h\}^{p+q-1} .
\end{aligned}
$$

Consider next the case when $p \geq 1$. Then the same function $f(x)$ is decreasing in $(m h,(n+1) h]$. Therefore,

$$
\begin{aligned}
h \sum_{l=m}^{n-1}\{(n-l) h\}^{p-1}\{(l-m+1) h\}^{q-1} \\
\quad \leq \int_{m h}^{n h} f(x) d x \leq \int_{m h}^{(n+1) h} f(x) d x \\
\quad=B(p, q)\{(n-m+1) h\}^{p+q-1} .
\end{aligned}
$$

Thus the lemma is proved.

Let us estimate the terms on the right hand side of (A.3) with the aid of this lemma. Since

$$
\begin{gathered}
h \sum_{l=m}^{n-k-2}\{(n-l) h\}^{(k+1) \beta-1}\{(l-m+1) h\}^{\alpha-1} \\
\leq B((k+1) \beta, \alpha)\{(n-m+1) h\}^{\alpha+(k+1) \beta-1} \\
h \sum_{l=l^{\prime}}^{n-k-2}\{(n-1-l) h\}^{(k+1) \beta-1}\left\{\left(l-l^{\prime}+1\right) h\right\}^{\beta-1} \\
\leq B((k+1) \beta, \beta)\left\{\left(n-l^{\prime}\right) h\right\}^{(k+2) \beta-1}
\end{gathered}
$$


(A.3) shows that (A.2) is true for $k+1$ also.

Thus (A.2) has been verified for every $0 \leq k \leq n-m$. In particular, when $k=n-m,(\mathrm{~A} .2)$ gives that

$$
x_{n, m} \leq a \Gamma(\alpha)\{(n-m+1) h\}^{\alpha-1} \sum_{j=0}^{n-m} \frac{\left[b \Gamma(\beta)\{(N+2) h\}^{\beta}\right]^{j}}{\Gamma(\alpha+j \beta)} .
$$

Since $\sum_{j=0}^{\infty}\left[b \Gamma(\beta)\{(N+2) h\}^{\beta}\right]^{j} / \Gamma(\alpha+j \beta)<\infty$, this shows the desired estimae.

By an analogous method we can prove also the following proposition.

Proposition A.3. The same assertion as in Proposition A.1 is true even if the inequality (A.1) is replaced by

$$
x_{n, m} \leq a\{(n-m+1) h\}^{\alpha-1}+h \sum_{l=m}^{n-1} x_{n, l+1} b\{(l-m+1) h\}^{\beta-1} .
$$

\section{A.2. Abstract evolution equations in a Banach space}

Let

$$
\left\{\begin{array}{l}
\frac{d u}{d t}+A u=f(t), \quad 0 \leq t \leq T \\
u(0)=u_{0}
\end{array}\right.
$$

be an equation in a Banach space $X$, where $-A$ is the generator of an analytic semigroup on $X$ (that is, $A$ satisfies the condition (A1) in Section 3 ), $f:[0, T] \rightarrow X$ is a given function, $u_{0} \in X$ is an initial value, and $u=u(t)$ is the unknown.

Let $e^{-t A}, 0 \leq t<\infty$, denote the semigroup generated by $-A$. As it is well known (for example, see [28, Theorem 3.4 in Chap. 3]), the solution $u$ is constructed by means of the semigroup.

Theorem A.4. Let $A$ satisfy (A1) in Section 3. Let $f \in \mathcal{C}^{\sigma}([0, T] ; X), 0<$ $\sigma<1$, and let $u_{0} \in \mathcal{D}(A)$. Then, (A.4) possesses a unique solution $u$ such that

$$
u \in \mathcal{C}([0, T] ; \mathcal{D}(A)) \cap \mathcal{C}^{1}([0, T] ; X)
$$

Moreover, the solution $u$ is given in the form

$$
u(t)=e^{-t A} u_{0}+\int_{0}^{t} e^{-(t-s) A} f(s) d s, \quad 0 \leq t \leq T .
$$

Furthermore, if we assume the compatibility conditions on $u_{0}$ and $f(t)$, then the solution $u$ enjoys higher regularity. Indeed, let $f \in \mathcal{C}^{p+\sigma}([0, T] ; X)$, where $p$ is 
a positive integer and $0<\sigma<1$, and let all the elements $u_{0}^{(1)}, \ldots, u_{0}^{(p)}$ such that

$$
\begin{aligned}
& u_{0}^{(1)}=-A u_{0}+f(0), \\
& u_{0}^{(k)}=-A u_{0}^{(k-1)}+f^{(k-1)}(0), \quad 2 \leq k \leq p,
\end{aligned}
$$

be well defined as elements belonging to $\mathcal{D}(A)$. Then, Theorem A.4 is applicable to

$$
\left\{\begin{array}{l}
\frac{d u^{(k)}}{d t}+A u^{(k)}=f^{(k)}(t), \quad 0 \leq t \leq T \\
u^{(k)}(0)=u_{0}^{(k)}
\end{array}\right.
$$

repeatedly for $k=1, \ldots, p$. As a consequence, we obtain that $u$ has the regularity

$$
u \in \mathcal{C}^{p}([0, T] ; X) \cap \mathcal{C}^{p-1}([0, T] ; \mathcal{D}(A))
$$

Consider next the non autonomous linear equation

$$
\left\{\begin{array}{l}
\frac{d u}{d t}+A(t) u=f(t), \quad 0 \leq t \leq T \\
u(0)=u_{0}
\end{array}\right.
$$

in $X$, where each $-A(t)$ is the generator of an analytic semigroup on $X$ with the domain $\mathcal{D}(A(t))$ independent of $t . A(t)$ are assumed to satisfy the Hölder condition (A3) announced in Section 4.

According to the Sobolevskii and Tanabe theory, a unique fundamental solution $U(t, s), 0 \leq s \leq t \leq T$, is constructed which gives the solution to (A.5).

Theorem A.5. Let $A(t)$ satisfy (A2) and (A3) in Section 4. Let $f \in$ $\mathcal{C}^{\sigma}([0, T] ; X), 0<\sigma<1$, and let $u_{0} \in \mathcal{D}(A(0))$. Then (A.5) possesses a unique solution $u$ such that

$$
u \in \mathcal{C}^{1}([0, T] ; X) \cap \mathcal{C}([0, T] ; \mathcal{D}(A(0)))
$$

Moreover, the solution $u$ is given in the form

$$
u(t)=U(t, 0) u_{0}+\int_{0}^{t} U(t, s) f(s) d s, \quad 0 \leq t \leq T
$$

with the unique fundamental solution $U(t, s), 0 \leq s \leq t \leq T$.

For the proof we refer the reader to Sobolevskii [25] or Tanabe [28]. 
As above we can obtain a more regular solution provided that the compatibility conditions on $u_{0}$ and $f(t)$ are satisfied. Indeed, let $A \in \mathcal{C}^{p-1+\mu}([0, T] ; \mathcal{L}(\mathcal{D}(A(0))$, $X))$ and $f \in \mathcal{C}^{p+\sigma}([0, T] ; X)$ with a positive integer $p$ and $0<\mu, \sigma<1$, and let all the elements $u_{0}^{(1)}, \ldots, u_{0}^{(p)}$ such that

$$
\begin{aligned}
& u_{0}^{(1)}=-A(0) u_{0}+f(0), \\
& u_{0}^{(k)}=-\sum_{l=0}^{k-1} A^{(k-l-1)}(0) u_{0}^{(l)}+f^{(k-1)}(0), \quad 2 \leq k \leq p
\end{aligned}
$$

be well defined as elements belonging to $\mathcal{D}(A(0))$. Then, the solution $u$ enjoys the regularity

$$
u \in \mathcal{C}^{p}([0, T] ; X) \cap \mathcal{C}^{p-1}([0, T] ; \mathcal{D}(A(0)))
$$

For the proof see [25].

Consider a quasilinear equation

$$
\left\{\begin{array}{l}
\frac{d u}{d t}+A(u) u=f(u), \quad 0 \leq t \leq T \\
u(0)=u_{0}
\end{array}\right.
$$

in $X$.

Here, $A(u)$ are defined for all $u \in K$, where $K$ is an open ball $K=\{u \in$ $Z$; $\left.\left\|u-u_{0}\right\|_{Z}<R\right\}$ of another Banach space $Z$ which is continuously embedded in $X$ and contains the initial value $u_{0}$, the domains $\mathcal{D}(A(u))$ are independent of $u \in K$. Each $-A(u)$ is the generator of an analytic semigroup on $X$. In addition, $A(u)$ are assumed to satisfy the Lipschitz condition (A5) announced in Section 5.

$f: K \rightarrow X$ is a given function satisfying the Lipschitz condition (F3) in Section 5. $u_{0}$ is an initial value of the problem, $u_{0}$ is assumed to satisfy $u_{0} \in \mathcal{D}\left(A\left(u_{0}\right)\right)$ and the condition (Sp) in Section 5.

Then the existence and uniqueness of local solution to (A.5) is already known.

TheOREm A.6. Let (A4-5), (Sp), (F3) and (In) in Section 5 be satisfied. Then, there exists a number $0<S \leq T$ such that on the interval $[0, S]$, (A.6) possesses a unique solution $u$ such that

$$
u \in \mathcal{C}\left([0, S] ; \mathcal{D}\left(A\left(u_{0}\right)\right)\right) \cap \mathcal{C}^{\eta}([0, S] ; Z) \cap \mathcal{C}^{1}([0, S] ; X)
$$

where $0<\eta<1-\alpha$ and $\alpha$ is the exponent appearing in (Sp).

For an outline of proof of this theorem, see [33]. For the detailed proof, see [16] or [25]. 


\section{References}

[ 1 ] G.A. Baker, J.H. Bramble and V. Thomée, Single step Galerkin approximations for parabolic problems, Math. Comp., 31 (1977), 818-847.

[ 2 ] N.A. Bobylev, J.K. Kim, S.K. Korovin and S. Piskarev, Semidiscrete approximations of semilinear periodic problems in Banach spaces, Nonlin. Anal., 33 (1998), 473-482.

[ 3 ] J.H. Bramble, A.H. Schatz, V. Thomée and L.B. Wahlbin, Some convergence estimates for semidiscrete Galerkin type approximations for parabolic equations, SIAM J. Numer. Anal., 14 (1977), 218-241.

[ 4 ] J.C. Butcher, The Numerical Analysis of Ordinary Differential Equations, John Wiley and Sons, Chichester, 1987.

[ 5 ] M. Crouzeix, S. Larsson, S. Piskarev and V. Thomée, The stability of rational approximations of analytic semigroup, BIT, 33 (1993), 74-84.

[6] H. Fujita and A. Mizutani, On the finite element method for parabolic equations, I; approximation of holomorphic semi-groups, J. Math. Soc. Japan, 28 (1976), 749-771.

[ 7 ] H. Fujita and T. Suzuki, Evolution Problems, Part 6 of Handbook of Numerical Analysis, Vol. II, Finite Element Methods (Part 1), edited by P.G. Ciarlet and J.L. Lions, NorthHolland, Amsterdam, 1991.

[ 8 ] H.-P. Helfrich, Fehlerabschätzungen für das Galerkinverfahlen zur lösung von Evolutionsgleichungen, Manuscripta Math., 13 (1974), 219-235.

[ 9 ] A. Iserles, A First Course in the Numerical Analysis of Differential Equations, Cambridge UP, New York, 1996.

[10] S.L. Keeling, Galerkin/Runge-Kutta discretizations for semilinear parabolic equations, SIAM J. Numer. Anal., 27 (1990), 394-418.

[11] E.F. Keller and L.A. Segel, Initiation of slime mold aggregation viewed as an instability, J. Theoret. Biol., 26 (1970), 399-415.

[12] S. Larsson, V. Thomée and L.B. Wahlbin, Finite-element methods for a strongly damped wave equation, IMA J. Numer. Anal., 11 (1991), 115-142.

[13] M.-N. Le Roux, Semidiscretization in time for parabolic problems, Math. Comp., 33 (1979), 919-931.

[14] Ch. Lubich and A. Ostermann, Runge-Kutta methods for parabolic equations and convolution quadrature, Math. Comp., 60 (1993), 105-131.

[15] Ch. Lubich and A. Ostermann, Runge-Kutta approximation of quasi-linear parabolic equations, Math. Comp., 64 (1995), 601-627.

[16] A. Lunardi, Abstract quasilinear parabolic equations, Math. Ann., 267 (1984), 395-415.

[17] A. Ostermann and M. Roche, Runge-Kutta methods for partial differential equations and fractional orders of convergence, Math. Comp., 59 (1992), 403-420.

[18] C. Palencia, A stability result for sectorial operators in Banach spaces, SIAM J. Numer. Anal., 30 (1993), 1373-1384.

[19] C. Palencia, Stability of rational multistep approximations of holomorphic semigroups, Math. Comp., 64 (1995), 591-599.

[20] A. Pazy, Semigroups of Linear Operators and Applications to Partial Differential Equations, Springer-Verlag, New York, 1983.

[21] S. Piskarev, On approximation of holomorphic semigroups, Tartu Riikl. Ül. Toimetised, 492 (1979), 3-32.

[22] P.H. Sammon, Convergence estimates for semidiscrete parabolic equation approximations, SIAM J. Numer. Anal., 19 (1982), 68-92.

[23] P.H. Sammon, Fully discrete approximation methods for parabolic problems with non- 
smooth initial data, SIAM J. Numer. Anal., 20 (1983), 437-470.

[24] G. Savaré, $A(\theta)$-stable approximations of abstract Cauchy problems, Numer. Math., 65 (1993), 319-335.

[25] P.E. Sobolevskiř, Equations of parabolic type in a Banach space, Amer. Math. Soc. Transl., 49 (1966), 1-62.

[26] T. Suzuki, An abstract study of Galerkin's method for the evolution equation $u_{t}+A(t) u=0$ of parabolic type with the Neumann boundary condition, J. Fac. Sci. Univ. Tokyo Sect. IA Math., 25 (1978), 25-46.

[27] T. Suzuki, Full-discrete finite element approximation of evolution equation $u_{t}+A(t) u=0$ of parabolic type, J. Fac. Sci. Univ. Tokyo Sect. IA Math., 29 (1982), 195-240.

[28] H. Tanabe, Equation of Evolution, Iwanami, Tokyo, 1975, (in Japanese); English translation, Pitman, London, 1979.

[29] T. Ushijima, Approximation theory for semi-groups of linear operators and its applications to approximation of wave equations, Japan J. Math., 1 (1975), 185-224.

[30] T. Ushijima, Approximation of semigroups and the finite element method, Sugaku (Japanese), 32 (1980), 133-148.

[31] A. Yagi, Fractional powers of operators and evolution equations of parabolic type, Proc. Japan Acad. Ser. A, 64 (1988), 227-230.

[32] A. Yagi, Parabolic evolution equations in which the coefficients are the generators of infinitely differentiable semigroups, Funkcial. Ekvac., 32 (1989), 107-124.

[33] A. Yagi, Abstract quasilinear evolution equations of parabolic type in Banach spaces, Boll. Un. Mat. Ital., 5-B (1991), 341-368.

[34] A. Yagi, Norm behavior of solutions to a parabolic system of chemotaxis, Math. Japon., 45 (1997), 241-265.

[35] K. Yosida. Functional Analysis, 6-th ed., Springer-Verlag, Berlin, 1980.

\author{
ETSUSHI NAKAGUCHI \\ Department Of ApPlied Physics \\ Graduate School Of EngineEring \\ OSAKA UNIVERSITY \\ 2-1, YAMADAOKA, SUITA \\ OSAKA 565-0871, JAPAN \\ E-mail: nakaguti@ap.eng.osaka-u.ac.jp \\ Atsushi Yagi \\ Department Of APPlied Physics \\ Graduate School Of Engineering \\ OSAKA UNIVERSITY \\ 2-1, YAMADAOKA, SUITA \\ OSAKA 565-0871, JAPAN \\ E-mai: yagi@ap.eng.osaka-u.ac.jp
}

